\title{
La volatilidad del tipo de cambio paralelo en Venezuela 2005-2015
}

\author{
The Volatility of the Parallel Exchange Rate \\ in Venezuela 2005-2015
}

\section{A volatilidade da taxa de câmbio paralela na Venezuela 2005-2015}

\author{
Laura Daniela Castillo Paredes* \\ Josefa Ramoni-Perazzi **
}




\section{Resumen}

El tipo de cambio paralelo constituye una de las principales variables económicas para la toma de decisiones en Venezuela. Para analizar el comportamiento de esta variable tomando en cuenta sus características inherentes, exceso de curtosis, persistencia y asimetría, se hace una síntesis teórica de los principales modelos estocásticos de volatilidad y se estima un conjunto de modelos. El modelo que mejor ajusta el comportamiento de la variable es un EGARCH $(1,1)$, que captura el efecto asimétrico de las perturbaciones estocásticas sobre la serie. Ante choques negativos (depreciación del tipo de cambio paralelo), la volatilidad asociada se incrementa, pero para choques positivos (apreciación del tipo de cambio paralelo), se mantiene constante.

Palabras clave: tipo de cambio paralelo, volatilidad, persistencia, modelos estocásticos de volatilidad, EGARCH.

Clasificación JEL: C1, C13, C22, C52, C53, F31

\section{Abstract}

The parallel exchange rate is one of the most important economic variables for decision making in Venezuela. With the purpose of analyzing the exchange rate considering its inherent characteristics, excess kurtosis, persistence and asymmetry, a theoretical synthesis of the main stochastic volatility models is made and a set of models is estimated. The results show that the model that best explains its behavior is an EGARCH (1.1); it captures the asymmetric effect of stochastic perturbations on the series. Negative shocks (depreciation of the parallel exchange rate) increase the volatility while positive shocks (appreciation of the parallel exchange rate) seem not to exert any effect.

Keywords: parallel exchange rate, volatility, persistence, stochastic volatility models, EGARCH. 


\section{Resumo}

A taxa de câmbio paralelo é uma das principais variáveis económicas para a tomada de decisões na Venezuela. Para analisar o comportamento desta variável tendo em conta as suas características essenciais, o excesso de curtose, persistência e assimetria, uma síntese teórica dos principais modelos de volatilidade estocástica é feita e um conjunto de modelos é estimado. O modelo que melhor se adapta ao comportamento da variável é um EGARCH (1.1), que capta o efeito assimétrico de perturbações estocásticas na série. Para choques negativos (depreciação da taxa de câmbio paralelo), a volatilidade aumenta, mas choques positivos (apreciação da taxa de câmbio paralelo) permanece constante.

Palavras-chave: taxa paralelo de câmbio, volatilidade, persistência, modelos de volatilidade estocástica, EGARCH. 


\section{INTRODUCCIÓN}

Ampliamente definido a nivel mundial, el tipo de cambio es el precio de una moneda en términos de otra. Expresa la cantidad de moneda doméstica que hay que pagar para adquirir una moneda extranjera (divisa), según el Banco Central de Venezuela (BCV, 2016), permitiendo el desarrollo de relaciones comerciales entre individuos, empresas o países. Es importante distinguir entre el tipo de cambio nominal (TCN), que para Samuelson y Nordhaus (2010) es el monto de moneda extranjera que se puede comprar con una unidad de moneda doméstica, y el tipo de cambio real (TCR) que, según Bernanke y Frank (2007), indica el precio medio de un bien o servicio en el interior del país, expresado en el precio medio de dicho bien o servicio en el mercado extranjero, lo que hace referencia a un precio relativo ${ }^{1}$.

En todas las economías abiertas del mundo, esta es una de las variables macroeconómicas más importantes y, desde el punto de vista econométrico, es una de las series de tiempo más interesantes. El sistema mediante el cual se determina el tipo de cambio que rige en la economía de un país puede ser de dos tipos: i) sistema de tipo de cambio flexible y ii) sistema de tipo de cambio fijo.

En el sistema de tipo de cambio flexible, se fija la paridad mediante las fuerzas de la oferta y la demanda de divisas en el mercado, donde participan todos los agentes económicos². Los países que

$1 \quad$ En este trabajo el tipo de cambio al que se hace referencia es el tipo de cambio nominal.

2 Este sistema se divide, a su vez, en sistema de flotación libre y sistema de flotación dirigida (flotación sucia). En el primero, el precio de la divisa de referencia es fijado estrictamente por el juego libre de las fuerzas de mercado y participan todos los agentes económicos sin ninguna restricción. En el segundo, el precio de la divisa es determinado por las fuerzas del mercado, pero el Estado interviene para influir en su cotización de tal manera que es el agente económico el que ejerce el mayor poder de fijación. 
cuentan con un sistema cambiario de tipo flexible tienen como consecuencias sobre sus economías: políticas monetarias independientes, necesidad de menos activos de reserva por parte de la autoridad monetaria, alta disciplina fiscal por parte del gobierno, además de ser un estabilizador automático de los choques de demanda; sin embargo, los tipos de cambio flexibles son más volátiles, esto afecta al comercio, demanda el desarrollo de mecanismos de cobertura y retrasa los ajustes en la asignación de recursos, lo que aumenta la variabilidad de la moneda local frente a la divisa de referencia.

En el sistema de tipo de cambio fijo, el Estado toma total control del mercado de divisas, dejando de lado las fuerzas de la oferta y la demanda; crea un mecanismo que permite vigilar el mercado, instaurando lo que se conoce como un control de cambio. Este no es más que la intervención oficial del mercado de divisas a través del diseño de un régimen administrativo, que siempre está acompañado por un conjunto de restricciones cuantitativas y cualitativas sobre la compra y venta de moneda extranjera. La fijación del tipo de cambio también es un instrumento de política cambiaria, con el fin único de controlar las entradas o salidas de capital, y su efectividad depende de las causas que han llevado a su implementación, de los objetivos planteados por los hacedores de política económica y la forma en que se implementa y opera en la práctica
(Dornbusch, Fischer \& Startz, 2009). Las consecuencias del control de cambio son básicamente: un estímulo para la disciplina monetaria ${ }^{3}$, reducir la especulación sobre la moneda nacional, proteger las reservas internacionales, la balanza de pago y el tipo de cambio nominal, la implementación de controles de precios sobre bienes, con la finalidad de prevenir la formación de un proceso inflacionario y regular automáticamente choques monetarios, permitiendo a los agentes económicos equilibrar sus tenencias de divisas porque transan moneda extranjera con la principal autoridad monetaria, esto busca que la volatilidad de la moneda local sea menor.

La era de la libre convertibilidad de las monedas permitió dejar atrás una serie de problemas asociados al control de la paridad cambiaria; el más conocido, la creación de un mercado paralelo de divisas. En este mercado se realizan operaciones de compra y venta de monedas extranjeras, la cotización en moneda nacional es fijada por el libre juego de la oferta y la demanda, pero al margen de la ley. A pesar de que el sistema cambiario fijo tiene como norte reducir la volatilidad de la moneda local respecto a la extranjera, el mercado paralelo que, por naturaleza, se crea, causa el efecto contrario, porque eleva la volatilidad del tipo de cambio nominal paralelo, distorsionando todo el entorno económico.

\footnotetext{
El precio de los excesos monetarios es la reducción de las reservas internacionales.
} 
La economía venezolana ha estado sometida a las rigurosidades de un excesivo control cambiario desde el año 2003, que maneja el mercado de divisas, tanto en términos de cantidad como de precio. Instaurado con la finalidad de evitar la fuga de capitales y la disminución de las reservas internacionales del país, las consecuencias que ha tenido la medida sobre el sistema económico han sido múltiples. La más conocida, el surgimiento de un mercado paralelo al mercado oficial, que deforma todo el aparato productivo de la economía, repercutiendo en la calidad de vida de los venezolanos. Esto ha hecho que una de las variables económicas de referencia para fijar precios de algunos bienes y servicios sea el tipo de cambio paralelo, porque el mercado no oficial cubre parte de la demanda de divisas. Durante el periodo que se estudia, la variación que ha sufrido el precio de la moneda extranjera en términos de la moneda nacional supera el $30500 \%$. Esta gran variabilidad despierta el interés de propios y extraños. Y es, precisamente, la característica en donde se enfoca este trabajo: la volatilidad del tipo de cambio paralelo.

El estudio de la volatilidad de las series de tiempo es un tema que durante años ha atraído el interés de muchos investigadores en esa área. Los últimos 35 años han marcado un antes y un después en la forma de abordar todo lo referente a este campo. Los desarrollos de Engle (1982), Bollerslev (1986) y Taylor (1986) fueron el punto de partida para estructurar un gigantesco y creciente marco teórico de referencia: la familia de los modelos autorregresivos de heterocedasticidad condicional (ARCH). Estos modelos se enfocan en analizar el comportamiento de la varianza condicional de la variable de interés, empleando métodos de estimación ampliamente conocidos; es allí donde radica su riqueza. Además, las series temporales de alta frecuencia que se observan en los mercados financieros y cambiarios vienen caracterizadas por ser asimétricas, leptocúrticas y con una elevada persistencia en volatilidad (Sánchez \& Reyes, 2006).

Es justamente la combinación de estos dos elementos: i) la volatilidad del tipo de cambio paralelo y ii) los modelos estocásticos de volatilidad disponibles para su análisis, lo que marca la hoja de ruta de la presente investigación. Con el objetivo de evaluar la conjunción de estos dos aspectos, se lleva a cabo la estimación de modelos estocásticos de volatilidad para 2869 observaciones de la variable de interés, y resalta, particularmente, la asimetría de las perturbaciones sobre la volatilidad del tipo de cambio paralelo y su persistencia sobre los valores futuros. Teniendo en mente la gama de modelos estadísticos existentes para estudiar las series de tiempo, desde el contexto uniecuacional, y la disponibilidad de herramientas computacionales, este estudio busca revelar el comportamiento de la variable tipo de cambio paralelo de Venezuela (TCP), mediante el uso de modelos estocásticos que tienen como principal componente la volatilidad de la serie. 
El artículo está organizado en cinco secciones, además de la introducción. La primera hace referencia a la evolución del tipo de cambio paralelo en Venezuela; la segunda muestra el marco teórico sobre los principales modelos estocásticos de volatilidad; la tercera sección describe la metodología utilizada; la cuarta sección ubica los resultados y las discusiones asociadas; y la quinta sección presenta las principales conclusiones encontradas.

\section{EVOLUCIÓN DE LOS TIPOS DE CAMBIO EN VENEZUELA 2003- 2015}

En los años 2001 y 2002 se registró una caída en las reservas internacionales venezolanas de 19,77\%, producto de la inseguridad política que vivía el país. En febrero del 2002, Venezuela mantuvo sus bandas cambiarias y el dólar se cotizó en $0,793 \mathrm{Bs} / \mathrm{USD}$ para la venta, hasta la entrada en vigencia de un esquema de flotación, que llevó el tipo de cambio a 0,981 Bs/USD para la venta, según cifras del BCV. El 5 de febrero de 2003 se instaura un control cambiario en el país y se crea la Comisión de Administración de Divisas (CADIVI). Durante este largo periodo se han establecido distintas instituciones con la finalidad de paliar los efectos del mercado paralelo. En junio de 2010 entró en vigencia el Sistema de Transacciones con Títulos en
Moneda Extranjera (SITME), dirigido a importaciones no prioritarias, utilizado de forma muy limitada por la población para ciertas operaciones, y se mantuvo hasta febrero de 2013. En marzo del año 2013 se creó el Sistema Complementario de Administración de Divisas (SICAD), su valor se fijaba por subastas pero los resultados no fueron los esperados. Para enero de 2014 CADIVI pasó a formar parte del Centro Nacional de Comercio Exterior (CENCOEX) y el país mantuvo un sistema de cambio dual. En febrero de 2014 el ente rector decidió modificar la Ley de Régimen Cambiario y sus ilícitos y crear el SICAD II, que funcionaba igual que el SICAD I, pero permitía el acceso a un mayor número de sectores económicos, incluyendo personas naturales. Para febrero de 2015 las autoridades venezolanas anunciaron el funcionamiento de un sistema cambiario de tres mercados: 1) CENCOEX; 2) SICAD (unificación del SICAD I y II) y, 3) Sistema Marginal de Divisas (SIMADI), donde el precio de dólar estadounidense fluctúa en función del libre juego de la oferta y la demanda.

En la Tabla 1 se puede apreciar la evolución de la moneda nacional después del establecimiento del control cambiario de 2003 y los sectores a quienes estaba dirigido; mecanismo de control que sigue vigente. 
Tabla 1. Evolución del control de cambio en Venezuela, 2003-2015

\begin{tabular}{|c|c|c|}
\hline Año & $\begin{array}{c}\text { Precio oficial del } \\
\text { dólar (Bs/USD) }\end{array}$ & Sector \\
\hline 2003 & 1,6 & Todos los sectores \\
\hline 2004 & 1,92 & Todos los sectores \\
\hline 2005 & 2,15 & Todos los sectores \\
\hline \multirow{3}{*}{2010} & 2,60 & Sectores prioritarios \\
\cline { 2 - 3 } & 4,30 & Resto de sectores \\
\cline { 2 - 3 } & 5,30 & SITME \\
\hline \multirow{2}{*}{2011} & 4,30 & Todos los sectores \\
\cline { 2 - 3 } & 5,30 & SITME \\
\hline \multirow{3}{*}{2013} & 6,30 & Todos los sectores \\
\cline { 2 - 3 } & 11,50 & SICAD \\
\cline { 2 - 3 } & 6,30 & CENCOEX \\
\cline { 2 - 3 } & 12,00 & SICAD I \\
\hline \multirow{3}{*}{2015} & 52,10 & SICAD II \\
\cline { 2 - 3 } & 6,30 & CENCOEX \\
\cline { 2 - 3 } & $13,50^{*}$ & SICAD \\
\hline \multirow{2}{*}{} & $199,53^{*}$ & SIMADI \\
\hline
\end{tabular}

* Precio del dólar al cierre del mes de noviembre.

El control de cambio vigente en Venezuela desde el año 2003 ha distorsionado toda la economía del país, por el aumento constante del tamaño del mercado paralelo, debido a las limitaciones en la asignación de divisas; reflejo claro es el comportamiento de los principales agregados macroeconómicos: la disminución de la tasa de crecimiento del producto interno bruto (PIB), el aumento de la tasa de inflación y la disminución de las reservas internacionales, por mencionar algunos, como se constata en la Tabla 2, que confirman la ineficiencia de la medida de política cambiaria. Desde entonces, el restringido mercado oficial coexiste con un mercado paralelo ilegal creciente.

Durante los primeros años existió una cotización paralela del dólar, denominada informalmente como el "dólar CANTV”, vigente hasta mayo del año 2008, cuando el Gobierno nacionalizó la Compañía Anónima Nacional Teléfonos de Venezuela (CANTV), eliminando toda posible operación cambiaria. Le siguió el "mercado permuta”, al que se accedía a través de bonos en moneda extranjera emitidos por el BCV, cuyo precio llegó casi a duplicar la tasa oficial vigente para

$4 \quad$ El nombre se originó de una de las principales acciones transadas en la Bolsa de Valores de Caracas, la de CANTV, la empresa cotizaba certificados de depósitos americanos (ADRs) en Estados Unidos, el agente económico (individuos o empresa) podía comprar acciones en Venezuela en bolívares, definirlas como ADRs, y venderlas en New York en dólares.

5 En este mercado, las personas que tenían bonos en bolívares y deseaban dólares, se los cambiaban a quienes tenían un bono denominado en dólares y querían bolívares, mediante la intermediación de las casas de bolsa, sumando las comisiones y las compensaciones de rigor. 
ese momento. En mayo de 2010 el Gobierno suspendió el mercado permuta debido a "irregularidades" en su funcionamiento, a pesar de que este mercado suministraba entre el $15 \%$ y $20 \%$ de las divisas requeridas por el sector privado, pero el diferencial entre el tipo de cambio oficial y el no oficial era cercano al $300 \%$, administrativamente inviable para las autoridades por las repercusiones sobre los precios.

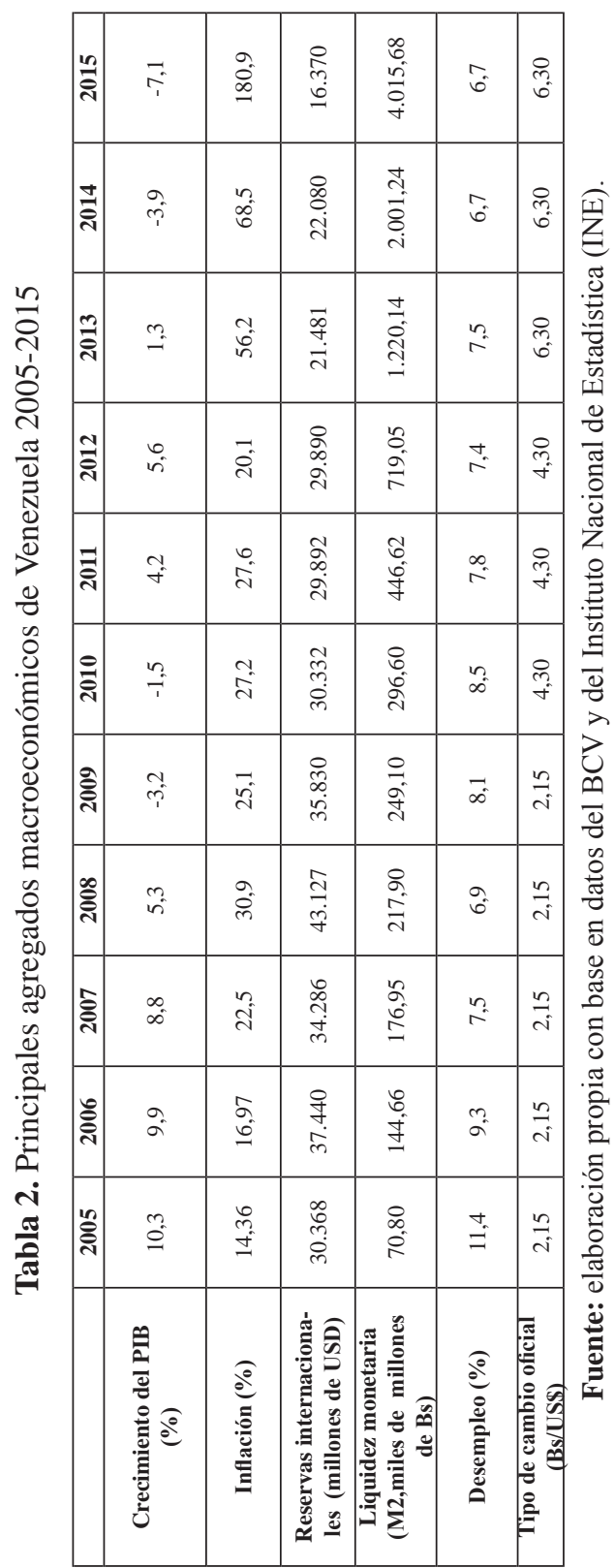


Después del cierre del mercado permuta, la determinación de la tasa del tipo de cambio paralelo se estableció con el llamado "dólar Cúcuta”, que toma en cuenta el tipo de cambio fronterizo en bolívar/peso (TCF) con la ciudad de Cúcuta (Colombia) a través de la oferta y demanda de bolívares en esa ciudad, y la información del BCV referente a la liquidez monetaria (M2) dividida entre las reservas internacionales (RRII) de Venezuela, es decir, el tipo de cambio implícito, para finalmente fijar como precio de referencia del dólar paralelo el promedio de los dos anteriores ${ }^{6}$.

Las presiones en el mercado paralelo han sido crecientes. La caída del precio del petróleo ha disminuido los ingresos del gobierno en dólares, recortando los cupos de divisas, lo que obliga a muchas personas e instituciones a acudir al mercado paralelo para conseguir los dólares que necesitan. Esto presiona la subida del tipo de cambio paralelo ampliando la brecha con el tipo de cambio oficial, que a su vez, presiona al Gobierno para que, tarde o temprano, devalúe el bolívar. Esta situación se convierte en un espiral negativo para la economía del país. Además, debe añadirse el hecho de que la cotización que se usa como marcadora es de frontera, con todas las limitaciones que ello implica; este tipo de cambio deja de lado las operaciones más refinadas que hace el sector corporativo venezolano; además, la dinámica fronteriza en Venezuela está fuertemente distorsionada por el fenómeno del contrabando; no obstante, el marcador de las operaciones de frontera, con sus imperfecciones, es la única referencia válida que se tiene sobre el tipo de cambio paralelo (Ecoanalítica, 2014).

En la Figura1 se puede apreciar la evolución del tipo de cambio del dólar paralelo frente al dólar oficial. La brecha creciente entre ambas cotizaciones estimuló la actividad especulativa y llevó a ajustar varias veces la tasa oficial hacia arriba, con la esperanza de disuadir el diferencial. Pero en la medida que se hacía más difícil adquirir divisas legalmente, mayor era la demanda en el mercado paralelo y, por lo tanto, mayor la diferencia entre el tipo de cambio de este mercado y el tipo de cambio oficial, hecho económico evidente.

$\mathrm{TCP}=\frac{T C F+(M 2 / R R I I)}{2}$ 


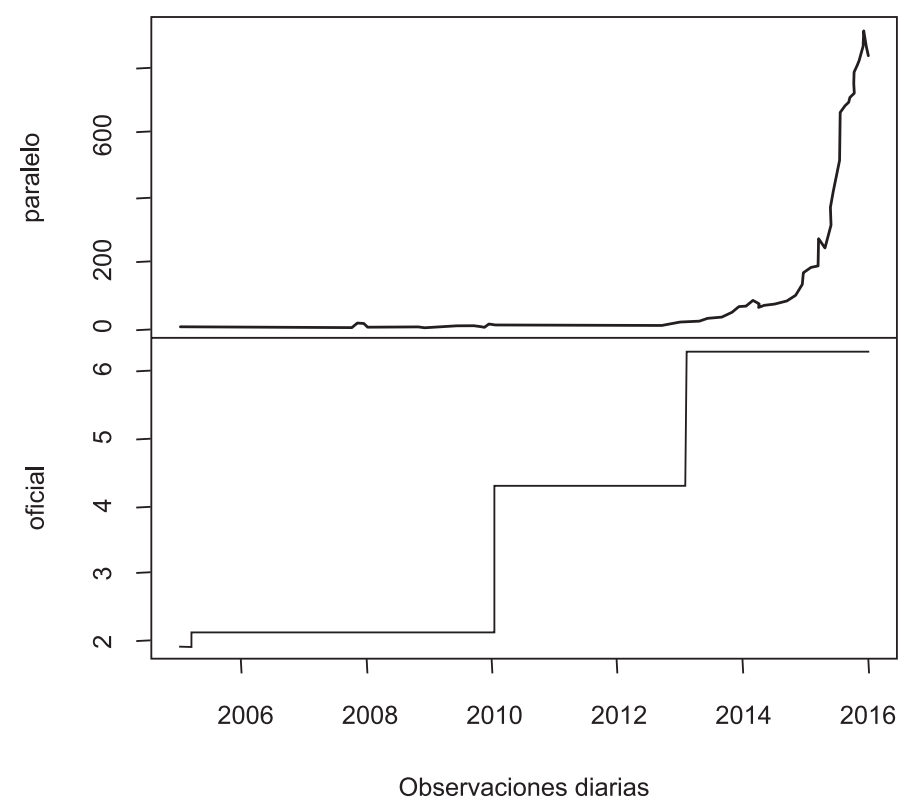

Figura 1. Tipo de cambio oficial versus tipo de cambio paralelo en Venezuela 2005-2015.

Fuente: elaboración propia con base en datos BCV y dólar Cúcuta.

Es importante destacar en este punto el crecimiento significativo que ha tenido la liquidez monetaria durante el periodo estudiado, como se observa en la Tabla 2. Esto permite suponer una fuerte relación entre la expansión monetaria y el aumento de la cotización del tipo de cambio paralelo, ambos con comportamiento creciente desbordado. Dicha relación se asume, tomando en consideración el famoso modelo de overshooting ${ }^{7}$ de Dornbusch (1976); modelo dinámico bajo expectativas racionales que intenta explicar la gran volatilidad de los tipos de cambio como consecuencia de un aumento inesperado en la cantidad de dinero (liquidez monetaria). Concretamente, ante un estímulo monetario el tipo de cambio sobrerreacciona, reflejando la diferencia de velocidades en el ajuste del mercado de activos financieros y el mercado de bienes. El tipo de cambio se ajusta rápidamente en el primer mercado, mientras que en el segundo lo hace lentamente. En consecuencia, toda expansión monetaria aumenta la liquidez, disminuye la tasa de interés y deprecia el tipo de cambio por encima de su nivel de equilibrio a largo plazo ${ }^{8}$.

No es objetivo de este trabajo contrastar la hipótesis del overshooting para el tipo de cambio paralelo en Venezuela.

8 Ante aumentos de la liquidez monetaria el tipo de cambio nominal sufre un proceso de ajuste. En el corto plazo se presenta una depreciación del tipo de cambio, generando alta volatilidad sobre éste y movimientos en los términos de intercambio. Durante ese proceso de ajuste, aumentan los precios en el mercado de bienes, lo que genera como consecuencia un efecto directo del tipo de cambio sobre la inflación y la demanda agregada de bienes nacionales. 


\section{MARCO TEÓRICO}

Las variables financieras y cambiarias tienen la particularidad de responder a los acontecimientos ocurridos con anterioridad, para determinar, en líneas generales, su comportamiento futuro. La volatilidad puede ser entendida como la relación existente entre el pasado y el presente de una serie de tiempo, medida por la magnitud de esa relación, tomada como una medida de riesgo de los activos financieros.

La volatilidad es una medida de la intensidad de los cambios aleatorios o impredecibles en la rentabilidad o en el precio de un título; en la representación gráfica de una serie histórica de rendimientos se asocia la volatilidad con la amplitud de las fluctuaciones del rendimiento tanto es que se consideren en valor absoluto como en desviaciones alrededor de un valor medio (Márquez, 2002)

No existe una definición concreta y generalizada de volatilidad, lo que sí es aceptado universalmente es que la volatilidad se refiere a un periodo en el cual la serie de tiempo presenta alta variabilidad o una varianza creciente (Baillie, 2006). Las características propias de una serie de tiempo con volatilidad son:
1. Exceso de curtosis (Mandelbrot, 1963; Fama, 1963).

2. Persistencia o conglomerado de volatilidad (Mandelbrot, 1963; Engle 1982). Los periodos de alta volatilidad son seguidos de periodos de volatilidad elevada, y los de baja volatilidad operan de forma análoga a corto plazo.

3. Existencia de discontinuidades en la serie (Figlewski, 1997), es decir, valores elevados de la serie en momentos específicos.

4. Comportamiento asimétrico de las series temporales, según la noticia que afecte la serie (Campbell, 1987; Hentschel, 1995).

5. Los períodos de alta o baja volatilidad son seguidos de períodos en los que la volatilidad es moderada a largo plazo (Hsieh, 1995; Figlewski, 1997).

En el estudio de las series de tiempo, la volatilidad se relaciona con la varianza condicional $^{9}$ de la serie. En general, esta última no es constante y, en consecuencia, los modelos de series de tiempo tradicionales, que suponen varianza homocedástica, no son adecuados para modelar este tipo de series. Es necesario asociar la varianza a una distribución de probabilidad concreta para poder utilizarla como medida del riesgo de activos financieros (Poon \& Granger, 2003).

$9 \quad$ Las medias y varianzas condicionales se asocian a los pronósticos a corto plazo y las medias y varianzas incondicionales se asocian a los pronósticos a largo plazo. 
La volatilidad permite estimar y medir el efecto de los cambios que no se pueden predecir; estudios sostienen que la volatilidad puede ser de dos fuentes: i) determinista, que no cambia a lo largo del tiempo y si cambia es de forma conocida; y ii) estocástica, que cambia a lo largo del tiempo de forma desconocida.

Los modelos estocásticos de volatilidad permiten modelar el comportamiento de la serie en estudio y predecir su comportamiento futuro con mayor precisión, se argumenta a favor:

- Miden los cambios de volatilidad de la serie en el tiempo.

- Proporcionan mejores estimaciones locales de la volatilidad.

- Capturan el agrupamiento de la volatilidad en el tiempo.

- Permiten estudiar la persistencia de la volatilidad.

- Facilitan las realizaciones de la volatilidad a corto plazo.

Existen diferentes modelos estocásticos de volatilidad, que con sus ventajas y desventajas forman una gama impresionante de herramientas disponibles para el modelado de series de tiempo.

Si bien, no es para nada despreciable el esfuerzo realizado por los investigadores que desarrollaron los modelos de volatilidad histórica y la metodología Box-Jenkins, son ampliamente conocidas las desventajas de dichos métodos. Estos modelos hablan de la estructura de la media condicional de los datos, el supuesto de varianza constante y la linealidad de dichos modelos hacen que se limite el estudio de algunas series temporales o se obtengan conclusiones erradas, viéndose afectada la eficiencia de los parámetros estimados. Es por esta razón que surgen en la década de los 80 una serie de modelos que se enfocan en estudiar los momentos condicionales de segundo orden de las series de tiempo ${ }^{10}$, conocidos modernamente como la familia de modelos autorregresivos condicionales heterocedásticos. En la Tabla 3 se encuentran los principales modelos de esta familia y en la Tabla 4 sus principales características.

\footnotetext{
$\overline{10} \quad$ El número de modelos derivados de estos estudios es considerable, en este trabajo solo se hará referencia a
} los más populares. 
Tabla 3. Principales modelos de la familia ARCH

\begin{tabular}{|c|c|c|}
\hline Modelo & Autor & Ecuaciones y Varianza $\left(h_{t}\right)$ \\
\hline $\begin{array}{l}\text { Modelo autorregre- } \\
\text { sivo condicional } \\
\text { heterocedástico } \\
{[\mathrm{ARCH}(q)]}\end{array}$ & $\begin{array}{l}\text { Engle } \\
\text { (1982) }\end{array}$ & $\begin{array}{l}y_{t}=\varepsilon_{t} \sqrt{ }\left(h_{t}\right) \\
h_{t}=\alpha_{0}+\sum_{i=1} \wedge q \alpha_{i} y_{t-i}^{2} \\
\varepsilon_{t}: \text { es un proceso ruido blanco, } \varepsilon_{t} \sim \text { iid }(0,1) \text {. Los parámetros deben } \\
\text { cumplir con la siguientes condiciones para alcanzar estacionariedad en } \\
\text { media: } \alpha_{0}>0 \text {, corresponde a la mínima varianza condicional observada; } \\
\alpha_{i} \geq 0 \forall i: 1,2, \ldots, q \text {, garantiza la existencia de varianza condicional e } \\
\text { incondicional positivas y } \sum_{\mathrm{i}=1}^{\mathrm{q}} \alpha_{i}<1 . \mathrm{Si} \varepsilon_{t} \text { es un ruido blanco gaussiano } \\
{\left[\varepsilon_{t} \sim \text { Niid }(0,1)\right] \text {, entonces } y_{t} \text { es condicionalmente normal y su varianza }} \\
\text { está dada por la ecuación [2]. }\end{array}$ \\
\hline $\begin{array}{l}\text { Modelo generalizado } \\
\text { autorregresivo con- } \\
\text { dicional heteroce- } \\
\text { dástico } \\
\text { [GARCH }(p, q)]\end{array}$ & $\begin{array}{c}\text { Bollerslev } \\
\text { (1986) }\end{array}$ & 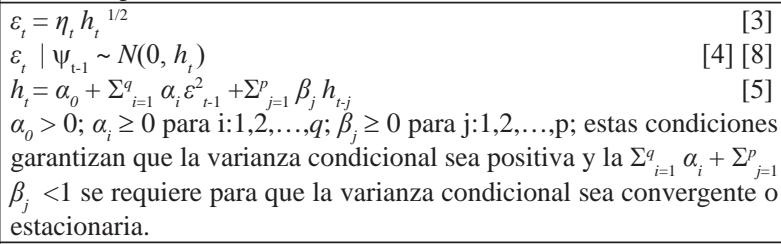 \\
\hline $\begin{array}{l}\text { Modelo generaliza- } \\
\text { do autorregresivo } \\
\text { condicional hetero- } \\
\text { cedástico integrado } \\
\text { (IGARCH) }\end{array}$ & $\begin{array}{l}\text { Engle y } \\
\text { Bollerslev } \\
(1986)\end{array}$ & $\begin{array}{l}\mathrm{E}_{t-1}\left(\varepsilon_{t}\right)=0 \\
\mathrm{E}_{t-1}\left(\varepsilon_{t}^{2}\right)=h_{t}=\alpha_{0}+\sum_{i=1}^{q} \alpha_{i} \varepsilon_{t-1}{ }^{2}+\sum^{p}{ }_{j=1} \beta_{j} h_{t-j} \\
\alpha_{0}>0 ; \alpha_{i} \geq 0 ; \beta_{j} \geq 0 \mathrm{y} 1-\sum_{i=1}^{q} \alpha_{i}+\sum^{p}{ }_{j=1} \beta_{j}=0 . \text { El proceso tiene } \mathrm{d}>0 \\
\text { raíces unitarias y el max }(p, q) \text {-d raíces fuera del círculo unitario, por } \\
\text { lo que el proceso es: } \\
\text { 1. Si } \alpha_{0}>0 \text { integrado en varianza de orden “d". } \\
\text { 2. Si } \alpha_{0}=0 \text { integrado en varianza con tendencia. }\end{array}$ \\
\hline $\begin{array}{l}\text { Modelo generalizado } \\
\text { autorregresivo con- } \\
\text { dicional heteroce- } \\
\text { dástico exponencial } \\
\text { [EGARCH }(p, q)]\end{array}$ & $\begin{array}{l}\text { Nelson } \\
(1991)\end{array}$ & $\begin{array}{l}\log \left(h_{t}\right)=\alpha_{0}+\sum_{i=1}^{q} \alpha_{i} g\left(\eta_{t-j}\right)+\Sigma^{p}{ }_{j=1} \beta_{j} \log \left(h_{t-j}\right) \\
g\left(\eta_{t}\right)=\theta \eta_{t}+\gamma\left[\left|\eta_{t}\right|-E\left(\left|\eta_{t}\right|\right)\right] \\
\text { donde, } \eta_{t} \text { es un ruido blanco, } \eta_{t} \sim i i d(0,1) \text {. i) la varianza condicional, } h \text {, } \\
\text { es lineal en } \eta_{t} \text {, cuya pendiente es } \alpha_{i}(\theta+\gamma) \text { cuando } \eta_{t}>0 \text { y es } \alpha_{i}(\theta-\gamma) \\
\text { cuando } \eta_{t}<0 \text {. Es este hecho el que genera el efecto asimétrico en la va- } \\
\text { rianza condicional. ii) el primer término de [9] permite una correlación } \\
\text { entre el error y futuras varianzas y iii) el segundo término de [9] es el } \\
\text { que produce el efecto ARCH. }\end{array}$ \\
\hline $\begin{array}{l}\text { Modelo generalizado } \\
\text { autorregresivo condi- } \\
\text { cional hetero-cedásti- } \\
\text { co con umbral } \\
{[\text { TGARCH }(p, q)]}\end{array}$ & $\begin{array}{c}\text { Zakoian } \\
\text { (1990) }\end{array}$ & 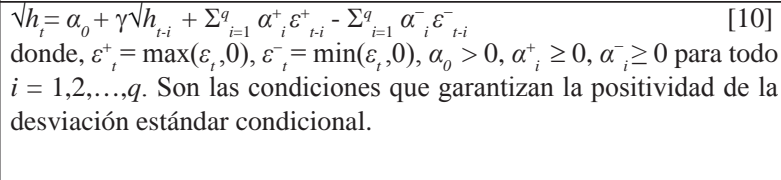 \\
\hline GJR-GARCH & $\begin{array}{l}\text { Glosten, Jagan- } \\
\text { nathan, } \\
\text { y Runkle } \\
\text { (1993) }\end{array}$ & $\begin{array}{l}y_{t}=\alpha_{0}+\alpha_{1}+h_{t-1}+\varepsilon_{t} \\
h_{t-1}=\alpha_{0}+\alpha_{1} \varepsilon_{t-1}^{2}+\alpha_{2} \varepsilon_{t-1}^{2} I_{t-1}+\beta h_{t-2} \\
I_{t-1}=\left\{0 \text { si } \varepsilon_{t-i}<0 \text { y } 1 \text { si } \varepsilon_{t-i}>0\right. \\
\log \left(h_{t-1}\right)=\alpha_{0}+\alpha_{1}\left(\eta_{t-1}^{2} /{ }_{h} h_{t-2}\right)+\alpha_{2}\left(\eta_{t-1}^{2} I_{t-1} / \sqrt{ } h_{t-2}\right)+\beta \log \left(h_{t-2}\right)\end{array}$ \\
\hline $\begin{array}{l}\text { AP-ARCH } \\
(p, q)\end{array}$ & $\begin{array}{l}\text { Ding, Granger } \\
\text { y Engle } \\
\text { (1993) }\end{array}$ & $\begin{array}{l}\text { El término }\left(\eta^{2}{ }_{t-1} / \sqrt{ } h_{t-2}\right) \text { minimiza el efecto de las subidas o bajadas de } \\
\text { la serie sobre los periodos posteriores. } \\
\varepsilon_{t}=\eta_{t} \sqrt{h}_{t} \\
\varepsilon_{t} \mid \psi_{t-1} \sim N\left(0, h_{t}\right) \\
h_{t}^{\delta}=\alpha_{0}+\sum_{i=1}^{q} \alpha_{1}\left(\left|\varepsilon_{t-1}\right|-\gamma_{i} \varepsilon_{t-1}\right)^{\delta}+\Sigma_{j=1}^{p} \beta_{j} h_{t-j}{ }^{\delta} \\
\text { donde, } \alpha_{0}>0 ; \delta \geq 0 ; \alpha_{0} \geq 0 \text {, para } i=1,2, \ldots, q \text { y } \beta_{j} \geq 0 \text {,para } j=1,2, \ldots, p \text {. }\end{array}$ \\
\hline
\end{tabular}


Tabla 4. Características de los principales modelos de la familia ARC

\begin{tabular}{|c|c|}
\hline Modelo & Características \\
\hline $\begin{array}{l}\text { Modelo autorregre- } \\
\text { sivo condicional } \\
\text { heterocedástico } \\
\text { [ARCH }(q)]\end{array}$ & $\begin{array}{l}\text { Fue el primer modelo formal que estudió el comportamiento de la varianza de una variable con } \\
\text { respecto al tiempo. Las características más sobresalientes de un modelo } A R C H(p) \text { son: i) las } \\
\text { esperanzas incondicional y condicional del proceso son nulas; ii) la varianza incondicional } \\
\text { es constante; iii) la varianza condicional no es constante, depende de los valores pasados } \\
\text { de la variable de estudio al cuadrado, es en sí misma un proceso autorregresivo (AR) que } \\
\text { genera errores heterocedásticos, condicionados por la varianza del error de periodos pasados; } \\
\text { iv) los errores estocásticos de la serie no presentan autocorrelación a nivel, pero no son } \\
\text { independientes porque se relacionan mediante sus varianzas; v) la estructura ARCH del } \\
\text { término de error y la estructura AR de la serie interactúan entre sí. Un shock grande en } y_{t} \text { se } \\
\text { asocia con una varianza persistentemente grande de los errores; a mayor valor del coeficiente } \\
\alpha_{i} \text { mayor será la persistencia. Mientas más alejada esté la serie } y_{t} \text { de su media, mayor es su } \\
\text { varianza y vi) la distribución incondicional de un proceso ARCH tiene forma desconocida. } \\
\text { Pero, se asegura que tiene colas más gruesas que las de una distribución normal, por ello } \\
\text { es leptocúrtica. En lo que respecta a la estimación de los parámetros de un modelo ARCH, } \\
\text { tal como lo planteó Engle, se realiza mediante el método de máxima verosimilitud (MV). }\end{array}$ \\
\hline $\begin{array}{c}\text { Dodelo } \\
\text { Moneralizado autorre- } \\
\text { gresivo condicional } \\
\text { heterocedástico } \\
\text { [GARCH }(p, q)]\end{array}$ & $\begin{array}{l}\text { Es una ampliación del modelo ARCH de Engle debido a las dificultades de estimación que } \\
\text { este presenta cuando se aplica a estructuras dinámicas de los cuadrados de las series, porque } \\
\text { se impone una estructura fija de rezagos. Esto implica que para estimar un ARCH se necesita } \\
\text { una estructura de rezagos muy larga de la varianza condicional para modelar la memoria de } \\
\text { los datos, hecho que genera una gran pérdida de grados de libertad. El aporte de Bollerslev } \\
\text { es fundamental en el desarrollo de los métodos de modelado de series de tiempo univariantes, } \\
\text { porque fortalece la utilidad del modelo ARCH. El modelo GARCH (p,q) permite capturar la } \\
\text { memoria larga de la serie y posee una estructura de rezagos más flexible. } \\
\text { Un modelo GARCH se destaca porque: i) } \eta_{t} \text { es un proceso estocástico estacionario } \\
\text { idénticamente distribuido con media cero y varianza unitaria, es decir, es un ruido blanco; ii) } \\
\text { la función de distribución marginal no es conocida pero se pueden calcular los primero dos } \\
\text { momentos del proceso; iii) el proceso } \varepsilon_{t} \text { es estacionario; iv) en los momentos condicionales, en } \\
t \text {, el valor de } t-1 \text { es una realización concreta conocida (no aleatoria); v) la varianza condicional } \\
\text { de las perturbaciones de la serie original constituyen un proceso ARMA, expresado por } h t \\
\text { y vi) los modelos GARCH son más parsimoniosos que los modelos ARCH con } q \text { grande, } \\
\text { lo que los hace más fáciles de identificar y de estimar. Bollerslev en su artículo definió el } \\
\text { término de la varianza condicional introducido en la ecuación [5] como un mecanismo de } \\
\text { aprendizaje adaptativo de un ARCH( } \infty \text { ). La estimación de los parámetros de un modelo } \\
\text { GARCH(p,q) se realiza por el método de MV. Para encontrar los estimadores MV Bollerslev } \\
\text { (1986) sugirió el uso de un algoritmo iterativo como el algoritmo de Berndt, Hall, Hall y } \\
\text { Hausman de } 1974 \text { (BHHH) y no el propuesto por Engle (1982), por la complejidad y la no } \\
\text { seguridad de convergencia. En } 1992 \text { Bollerslev y Wooldrige propusieron una estimación } \\
\text { cuasi máximo verosímil (CMV). }\end{array}$ \\
\hline $\begin{array}{c}\text { Modelo } \\
\text { generalizado autorre- } \\
\text { gresivo condicional } \\
\text { heterocedástico } \\
\text { Integrado } \\
\text { (IGARCH) }\end{array}$ & $\begin{array}{l}\text { Puede considerarse una variante entre un ARCH(q) y un GARCH(p,q) cuya característica } \\
\text { principal es estimar la varianza partiendo del hecho de que esta es integrada en varianza. En } \\
\text { los modelo IGARCH la información existente en el momento t es sumamente importante } \\
\text { para realizar predicciones óptimas en cualquier momento } t+s \text { de interés. El efecto sobre } h_{t+s} \\
\text { de } h_{t+1} \text { a medida que } s \rightarrow \infty \text { es cada vez menor pero nunca nulo, por lo que el efecto de la } \\
\text { varianza persiste sobre el modelo. } \\
\text { Engle y Bollerslev encontraron que la distribución de la varianza del error estocástico } \\
\text { presentaba una cola superior a la de una distribución normal, asumida para explicar los } \\
\text { modelos descritos en apartados anteriores. Ellos descubrieron que la verdadera distribución } \\
\text { que sigue la varianza es una t-student estandarizada La estimación por MV de los modelos } \\
\text { ARCH y la cuasi máximo verosímil de los GARCH son perfectamente aplicables a los } \\
\text { modelos IGARCH. }\end{array}$ \\
\hline
\end{tabular}




\begin{tabular}{|c|c|}
\hline Modelo & Características \\
\hline $\begin{array}{c}\text { Modelo } \\
\text { generalizado autorre- } \\
\text { gresivo condicional } \\
\text { heterocedástico ex- } \\
\text { ponencial [EGARCH } \\
\qquad(p, q)]\end{array}$ & $\begin{array}{l}\text { En } 1987 \text { Milhoj propuso un modelo ARCH logarítmico, debido a las dificultades encontradas al } \\
\text { estimar el modelo ARCH porque los coeficientes resultaban negativos. El modelo de la ecuación [8] } \\
\text { no requiere condiciones para asegurar que la varianza condicional sea positiva, pero una limitación } \\
\text { es que la varianza condicional } h_{t} \text { es simétrica en los valores pasados, por ende no pondera de } \\
\text { forma correcta la volatilidad asociada. Nelson (1991) estudió los modelos ARCH y GARCH, } \\
\text { y concluyó: i) las condiciones impuestas sobre los parámetros del modelo para asegurar la } \\
\text { positividad de la varianza condicional frecuentemente son incumplidas. Además, parámetros } \\
\text { siempre positivos generan estimaciones incorrectas por el efecto creciente que permanece } \\
\text { en la estimación, lo que evita la posibilidad de predecir un comportamiento oscilatorio; ii) } \\
\text { el modelo GARCH tiene incapacidad para modelar la asimetría de la volatilidad asociada } \\
\text { a las altas y bajas asociadas a la serie, porque la función de varianza condicional simétrica } \\
\text { es inapropiada puesto que no sería capaz de representar la correlación negativa entre la } \\
\text { volatilidad y los datos pasados; iii) refiriéndose a los modelos ARCH, Nelson (1991) declara } \\
\text { que tienen la debilidad de no poder medir la persistencia de un shock pasado sobre los valores } \\
\text { de la varianza condicional, por lo que si este impacto es persistente pasaría a formar parte } \\
\text { de la estructura temporal de la serie (Poterba \& Summers, 1986). No medir adecuadamente } \\
\text { su impacto altera las decisiones asociadas. }\end{array}$ \\
\hline $\begin{array}{l}\text { Modelo } \\
\text { generalizado autorre- } \\
\text { gresivo condicional } \\
\text { heterocedástico } \\
\text { con umbral } \\
\text { [TGARCH }(p, q)]\end{array}$ & $\begin{array}{l}\text { Zakoian, en } 1990 \text { llegó a derivar una clase de modelos en donde el interés residía sobre la } \\
\text { desviación estándar condicional. Al modelo descrito en [10] lo denominó el "threshold ARCH" } \\
\left(\text { TARCH), debido a que el coeficiente } \varepsilon_{t-1} \text { cambia cuando cruza el umbral del cero. Cuando }\right. \\
\varepsilon_{t-1}>0, \sqrt{h} \text { es lineal cuya pendiente es } \alpha_{i}^{+} \text {; por el contrario si } \varepsilon_{t-1}<0, \sqrt{ } h_{t} \text { sigue siendo lineal } \\
\text { pero la pendiente es } \alpha_{i}^{-} \text {. Es precisamente esta característica la que permite la asimetría en la } \\
\text { varianza condicional, comportamiento similar al del modelo EGARCH. Zakoian realizó una } \\
\text { extensión de su modelo añadiendo las desviaciones estándar condicionales pasadas como } \\
\text { variables explicativas. }\end{array}$ \\
\hline GJR-GARCH & $\begin{array}{l}\text { Los investigadores Glosten, Jagannathan y Runkle (1993), basados en los modelos } \\
\text { descubiertos hasta ese momento, desarrollaron un modelo ARCH para comprobar ciertas } \\
\text { características de los mercados bursátiles, específicamente. Suponiendo que el modelo sigue } \\
\text { una estructura ARCH, en donde el presente se alimenta de información pasada, genera dos } \\
\text { tipos de errores: uno propio del modelo y otro propio del método de estimación. El modelo } \\
\text { GJR-ARCH tiene tres características fundamentales: i) asimetría en la respuesta de la variable } \\
\text { ante las buenas y malas noticias. Las malas noticias generan con frecuencia mayor volatilidad } \\
\text { en la serie; ii) la volatilidad en los mercados bursátiles es inversamente proporcional a la } \\
\text { prima de riesgo de estos y iii) patrones estacionales en el comportamiento de la serie hacen } \\
\text { que se pueda modelar la misma incluyendo variables ficticias. }\end{array}$ \\
\hline $\begin{array}{c}\text { AP-ARCH } \\
\quad(p, q)\end{array}$ & $\begin{array}{l}\text { En 1993, Ding, Granger y Engle, basados en características que ellos encontraron comunes en } \\
\text { las series de tiempo financieras, propusieron: i) no solo existe autocorrelación entre los valores } \\
\text { de la perturbación estocásticas en términos absolutos, sino que esa perturbación elevada a } \\
\text { una potencia "d", muestra mayores autocorrelaciones; ii) no existen razones suficientes para } \\
\text { admitir, desde un principio, la relación lineal en la especificación de la varianza de los modelos } \\
\text { de la familia ARCH, respecto a los errores rezagados al cuadrado o a la desviación estándar } \\
\text { rezagada y iii) como la autocorrelación observada en las series financieras, evidencia ser } \\
\text { mayor en los valores cercanos a la unidad, no resulta correcto usar la varianza o la desviación } \\
\text { estándar, sino que un valor de esta última elevado a una potencia " } \delta \text { ”, que será determinada. }\end{array}$ \\
\hline \multicolumn{2}{|c|}{$\begin{array}{l}\text { Nota: existen otros modelos estocásticos de volatilidad como el LGARCH, que es una linealización del GARCH-M, } \\
\text { MGARCH consiste en especificar la varianza de forma multiplicativa, FACTOR ARCH que emplea la covarianza entre } \\
\text { varias series temporales, modelos ARCH de régimen cambiante, el Q-GARCH que es un modelo GARCH cuadrático, } \\
\text { y las diferentes variantes de los modelos ARCH y GARCH multivariante, pero no serán tratados en este estudio. }\end{array}$} \\
\hline
\end{tabular}




\section{METODOLOGÍA}

Este estudio es de carácter descriptivoexplicativo. Descriptivo porque busca detallar un fenómeno económico en una circunstancia temporal y geográfica determinada. Explicativo, ya que su finalidad es exponer el comportamiento de una variable, en este caso el tipo de cambio paralelo mediante el uso de modelos estadísticos; requiere de control tanto metodológico como estadístico. La investigación es documental, "aquella que se basa en la obtención y análisis de datos provenientes de materiales impresos u otro tipo de documentos" (Arias, 2006).

Los datos: se toma como fuente de datos la serie correspondiente al dólar paralelo con referencia al "dólar Cúcuta”, entre el 3 de enero de 2005 y el 1 de enero de 2016, con operaciones durante cinco días a la semana, por lo que sábados y domingos no están incluidos. Conformada por 2869 observaciones.

Haciendo referencia a la Figura 1, el comportamiento al alza de la serie puede explicarse, en parte, por el exceso de trabas burocráticas para el acceso a dólares oficiales, que por ley natural del mercado, ante escasez de oferta y exceso de demanda, impulsa el aumento del precio. Tal como era de esperarse, por ser una serie financiera, el tipo de cambio paralelo es leptocúrtica. En la Tabla 5 se detallan las estadísticas descriptivas del tipo de cambio paralelo.

Tabla 5. Estadísticas descriptivas del tipo de cambio paralelo de Venezuela 2005-2015

\begin{tabular}{|c|c|c|c|c|c|c|c|c|c|c|}
\hline Min. & 1stQu. & Median & Mean & 3rdQu. & Max. & Var & SD & Asimetría & Curtosis & Obs. \\
2,51 & 4,10 & 7,92 & 63,81 & 23,20 & 920,60 & 28260,9 & 168,10 & 3.636 & 12.61 & 2869 \\
\hline
\end{tabular}

Fuente: elaboración propia con base en datos dólar Cúcuta.

Se concluye para siete de siete pruebas, como se muestra en la Tabla 6, que el tipo de cambio paralelo de Venezuela no se ajusta a una distribución normal. Este problema se trató de corregir a través de transformaciones en logaritmo, la diferencia y los rendimientos, sin mayores resultados, aun cuando este último permite evidenciar la volatilidad de la serie, como se aprecia en la Figura 2 y en la Tabla 7. 
Tabla 6. Pruebas de normalidad del tipo de cambio paralelo

\begin{tabular}{|l|l|l|}
\hline \multicolumn{1}{|c|}{ Estadístico } & \multicolumn{1}{c|}{ Valor } & \multicolumn{1}{c|}{ P-value } \\
\hline Shapiro-Wilk & $\mathrm{W}=0,39755$ & $<2,2 \mathrm{e}-16$ \\
Jarque Bera & $\mathrm{X}$-squared $=25389$ & $<2,2 \mathrm{e}-16$ \\
Anderson-Darling & $\mathrm{A}=699.23$ & $<2,2 \mathrm{e}-16$ \\
Cramer-von Mises & $\mathrm{W}=141.98$ & $7.37 \mathrm{E}-10$ \\
Pearson & $\mathrm{P}=37361$ & $<2.2 \mathrm{e}-16$ \\
Lilliefors (Kolmogorov-Smirnov) & $\mathrm{D}=0.35769$ & $<2.2 \mathrm{e}-16$ \\
Shapiro-Francia & $\mathrm{W}=0.39744$ & $<2.2 \mathrm{e}-16$ \\
\hline
\end{tabular}

Fuente: elaboración propia con base en datos dólar Cúcuta. $\alpha=5 \%$.

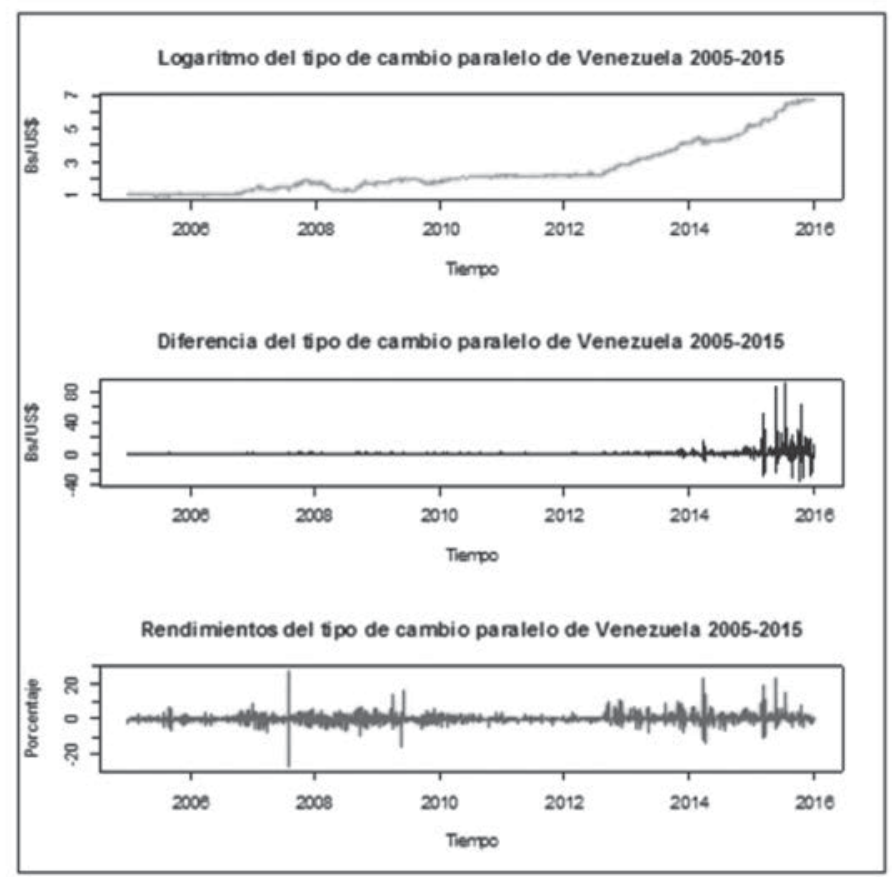

Figura 2. Transformaciones del tipo de cambio paralelo en Venezuela. 
Tabla 7. Pruebas de normalidad de las transformaciones del tipo de cambio paralelo

\begin{tabular}{|l|l|l|l|l|l|c|}
\hline & \multicolumn{2}{|c|}{ Logaritmo TCP } & \multicolumn{2}{c|}{ Diferencia TCP } & \multicolumn{2}{c|}{ Rendimientos TCP } \\
\hline \multicolumn{1}{|c|}{ Estadístico } & \multicolumn{1}{|c|}{ Valor } & \multicolumn{1}{c|}{ P-value } & \multicolumn{1}{c|}{ Valor } & \multicolumn{1}{c|}{ P-value } & Valor & P-value \\
\hline Shapiro-Wilk & $\mathrm{W}=0,83252$ & $<2,2 \mathrm{e}-16$ & $\mathrm{~W}=0,22728$ & $<2,2 \mathrm{e}-16$ & $\mathrm{~W}=0,73022$ & $<2,2 \mathrm{e}-16$ \\
\hline Jarque Bera & $\mathrm{X}=849,07$ & $<2,2 \mathrm{e}-16$ & $\mathrm{X}=3546500$ & $<2,2 \mathrm{e}-16$ & $\mathrm{X}=164280$ & $<2,2 \mathrm{e}-16$ \\
\hline Anderson-Darling & $\mathrm{A}=170,2$ & $<2,2 \mathrm{e}-16$ & $\mathrm{~A}=810,05$ & $<2,2 \mathrm{e}-16$ & $\mathrm{~A}=810,05$ & $<2,2 \mathrm{e}-16$ \\
\hline Cramer-von Mises & $\mathrm{W}=31,349$ & $7,37 \mathrm{E}-10$ & $\mathrm{~W}=173,7$ & $7,37 \mathrm{E}-10$ & $\mathrm{~W}=35,538$ & $7,37 \mathrm{E}-10$ \\
\hline Pearson & $\mathrm{P}=3845,9$ & $<2,2 \mathrm{e}-16$ & $\mathrm{P}=55020$ & $<2,2 \mathrm{e}-16$ & $\mathrm{P}=4942,7$ & $<2,2 \mathrm{e}-16$ \\
\hline $\begin{array}{l}\text { Lilliefors (Kolmogorov } \\
\text {-Smirnov) }\end{array}$ & $\mathrm{D}=0,24852$ & $<2,2 \mathrm{e}-16$ & $\mathrm{D}=0,40234$ & $<2,2 \mathrm{e}-16$ & $\mathrm{D}=0,16559$ & $<2,2 \mathrm{e}-16$ \\
\hline Shapiro-Francia & $\mathrm{W}=0,83297$ & $<2,2 \mathrm{e}-16$ & $\mathrm{~W}=0,22501$ & $<2,2 \mathrm{e}-16$ & $\mathrm{~W}=0,72737$ & $<2,2 \mathrm{e}-16$ \\
\hline
\end{tabular}

Fuente: elaboración propia con base en datos dólar Cúcuta. $\alpha=5 \%$.

En consecuencia, se decidió trabajar con los rendimientos del tipo de cambio paralelo (RTCP), porque tienen propiedades deseables para su modelado. Dicha serie, construida a partir de la expresión [17], obedece a la literatura consultada:

$$
R C T P=\left[\log \left(T C P_{\mathrm{t}}\right)-\log \left(T C P_{\mathrm{t}-1}\right)\right] * 100
$$

La serie RTCP no se distribuye normal, presenta un fuerte apuntamiento y resulta estacionaria enmedia. Adicionalmente, no es independiente y está correlacionada, según los resultados del test de independencia de Brock, Dechert y Scheinkmanes (BDS), es decir, no es ruido blanco, como se confirma en la Tabla 8 y en la Figura 3.

Tabla 8. Estadísticas de los rendimientos del tipo de cambio paralelo de Venezuela, 2005-2015

\begin{tabular}{|c|c|c|c|c|c|c|c|c|c|c|}
\hline \multicolumn{11}{|c|}{ Estadística Descriptiva } \\
\hline \multirow{2}{*}{\begin{tabular}{|c|c|} 
Min. & 1 stQu. \\
$-27,650$ & $-0,389$
\end{tabular}} & \begin{tabular}{l|l} 
I. & Median
\end{tabular} & Mean & 3rdQu & Max. & Var & SD & \multicolumn{2}{|c|}{ Asimetría } & Curtosis & Obs. \\
\hline & 0,000 & 0,1996 & 0,7380 & 28,100 & 4,6860 & 2,164 & \multicolumn{2}{|c|}{1,42330} & 36,9397 & 2868 \\
\hline \multicolumn{11}{|c|}{$\begin{array}{l}\text { Pruebas de Dickey-Fuller Aumentada (ADF) y } \\
\text { Phillis - Perron (PP) para raíz unitaria }\end{array}$} \\
\hline $\mathrm{ADF}$ & $-12,376$ & \multicolumn{2}{|c|}{\begin{tabular}{|l|l}
$\operatorname{Prob}(A D F)$ \\
\end{tabular}} & 0,01 & $\mathrm{PP}$ & \multicolumn{2}{|c|}{$-2936,2$} & \multicolumn{2}{|c|}{ Prob(PP) } & 0,01 \\
\hline \multicolumn{11}{|l|}{ Test BDS } \\
\hline Dimensión & \multicolumn{3}{|c|}{ Estadístico BDS } & \multicolumn{2}{|c|}{ Std. Error } & \multicolumn{3}{|c|}{ z-Estadístico } & \multicolumn{2}{|c|}{ Prob. } \\
\hline 2 & \multicolumn{3}{|c|}{0.037699} & \multicolumn{2}{|c|}{0.002508} & \multicolumn{3}{|c|}{15.02963} & \multicolumn{2}{|c|}{0.0000} \\
\hline 3 & \multicolumn{3}{|c|}{0.069207} & \multicolumn{2}{|c|}{0.003998} & \multicolumn{3}{|c|}{17.30827} & \multicolumn{2}{|c|}{0.0000} \\
\hline 4 & \multicolumn{3}{|c|}{0.09349} & \multicolumn{2}{|c|}{0.004779} & \multicolumn{3}{|c|}{19.56363} & \multicolumn{2}{|c|}{0.0000} \\
\hline 5 & \multicolumn{3}{|c|}{0.107833} & \multicolumn{2}{|c|}{0.005} & \multicolumn{3}{|c|}{21.56468} & \multicolumn{2}{|c|}{0.0000} \\
\hline 6 & & 0.11456 & & 0.00 & 4842 & & 6590 & & 0.00 & \\
\hline
\end{tabular}

Fuente: elaboración propia con base en datos dólar Cúcuta. $\alpha=5 \%$. 


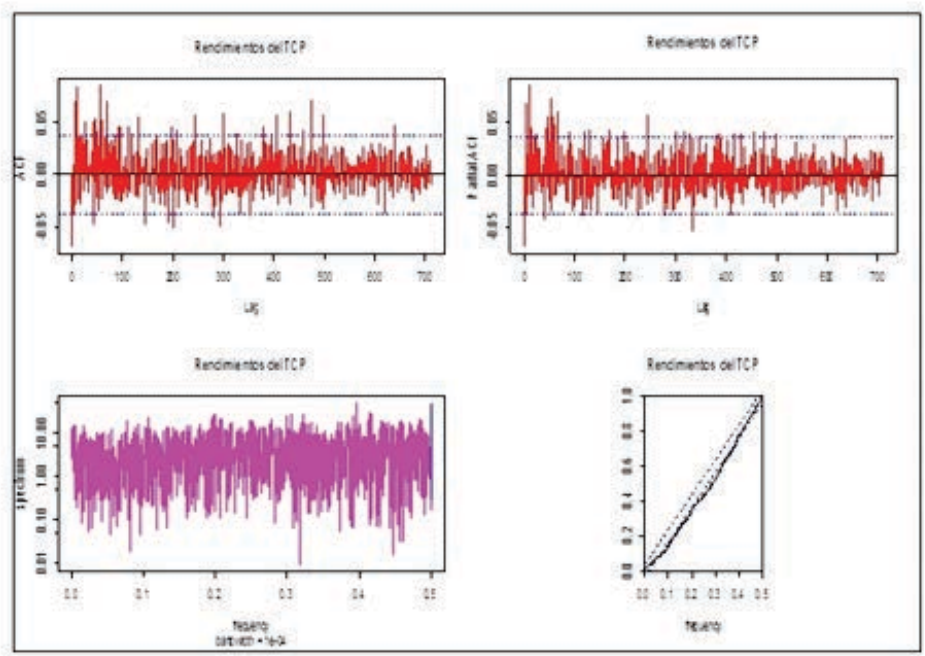

Figura 3. AFC, PACF y periodograma acumulado de RTCP

Así, los rendimientos del tipo de cambio paralelo concuerdan con las características planteadas por Mandelbrot (1963) y Fama (1963) para las series financieras:

- Concentración de los datos en torno a la media.

- Distribución leptocúrtica (apuntamiento de las observaciones).

- Colas gruesas de la distribución, aunque no muy evidente.

- En concreto se estiman modelos ARCH, GARCH, EGARCH, GJRGARCH y AP-ARCH para el tipo de cambio paralelo, siguiendo la metodología de sus autores, descrita anteriormente.

Los modelos: el método de estimación de los parámetros de interés que se usa es el de máximo verosimilitud (MV) para los modelos ARCH y AP-ARCH, haciendo caso a la afirmación de diversos autores (Engle, Bollerslev, Nelson, entre otros) que es un método asintóticamente superior al método de mínimos cuadrados (MCO), por lo que logra estimadores eficientes, dada la ausencia de linealidad ${ }^{11}$.

Los modelos GARCH, EGARCH y GJRGARCH se estiman mediante el método de cuasi máximo verosimilitud (CMV), usado con menos frecuencia que el MV, pero empleado cuando no se cumple el supuesto de normalidad de las variables en estudio ${ }^{12}$.

El algoritmo utilizado para estimar los modelos es el Berndt, Hall, Hall y Hausman

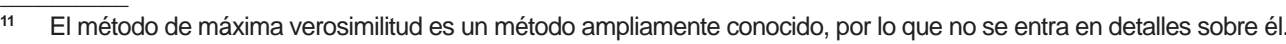

12 El método de CMV garantiza que los parámetros estimados sean consistentes para la parametrización conjunta de la media y la varianza condicionales. Ver el apéndice $n .^{\circ} 2$.
} 
(BHHH, 1974). Este, a diferencia de los métodos de estimación tradicional que asumen linealidad en variables y parámetros, supone la existencia de relación no lineal en ambos, parámetros y variables ${ }^{13}$, además de garantizar la convergencia de los valores estimados.

En cuanto al software, el procesamiento de los datos y la estimación de los modelos se realiza con The $R$ Project for Statistical Computing, versión 3.3.1, los programas, modificados, se basan en los suministrados por el sitio web y las librerías electrónicas asociadas.

\section{RESULTADOS Y DISCUSIÓN}

En concreto, dadas las consideraciones del marco teórico, se procedió a estimar los modelos, se concentró el trabajo en la rama de los modelos no lineales (modelos de la familia ARCH). Después de esto, se observaron los siguientes resultados:

1. Examinados los cuadros de los rendiemientos del tipo de cambio paralelo ${ }^{14}$, Figura 4, estos concordaron con los hallazgos hechos por Ding et al. (1993) sobre el índice S\&P 500:

1. Tendencia creciente de la serie sin transformar, acentuada al final del periodo, producto de la alta volatilidad.

11. Los rendimientos del tipo de cambio paralelo eliminan la tendencia, porque se distribuye de manera estable en torno a la media que es 0,1996.

111.El valor absoluto de los rendimientos refleja el carácter volátil de la serie, confirmando la persistencia de esta o los conglomerados de volatilidad que exponían Mandelbrot (1963) y Engle (1982).

2. La estructura de autocorrelación de la serie ajustó a través de un proceso de autocorrelación de orden uno, como lo estimaran Ding et al. en 1993. En la Tabla 9, los valores negativos de los coeficientes de autocorrelación reflejaron la recuperación de los rendimientos, que se conoce en la literatura como "recuperación de beneficios o reversión media". En el caso de los rendimientos al cuadrado y sus valores absolutos se cumplió que son todos estadísticamente significativos, este hecho puso de manifiesto dos cosas:

1. La existencia de una memoria larga en la serie.

it. La persistencia temporal de la volatilidad asociada, es decir, presencia de conglomerados de volatilidad.

\footnotetext{
Para una explicación teórica del algoritmo, ver el apéndice $n .^{\circ} 3$.

14 Porque se tomó en consideración las sugerencias de Engle y Bollerslev (1986), ya que son estos los que corresponden con el valor de la varianza.
} 


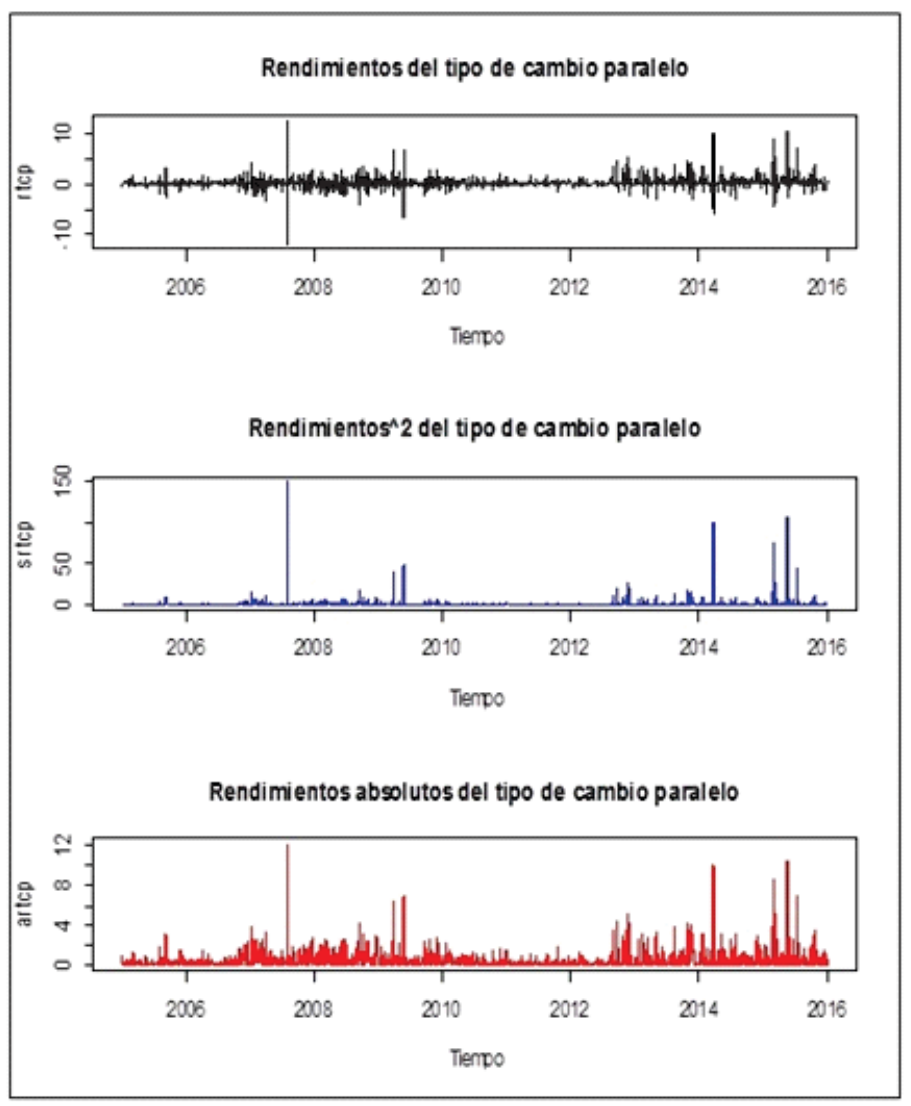

Figura 4. Rendimientos del TCP 

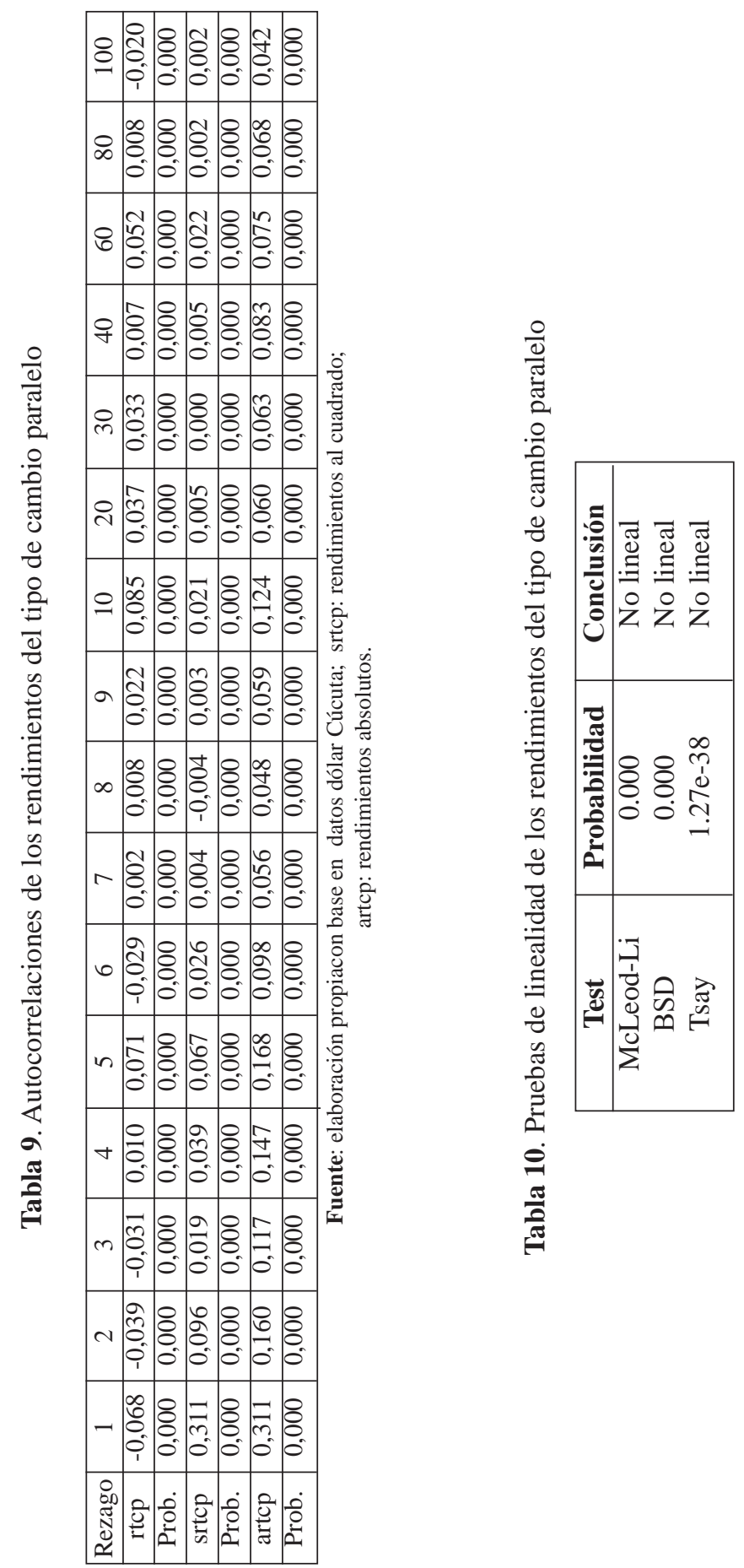
3. La presencia de estructuras no lineales en la serie se confirmó con las pruebas linealidad mostradas en la Tabla 10 y con la estimación de un modelo de regresión lineal, que incluyó una estructura promedio móvil de orden uno $[M A(1)]$ de la forma

$$
r t c p_{t}=\hat{\beta}_{1}+\hat{\beta}_{2} \varepsilon_{t-1}+\hat{\varepsilon}_{t}
$$

mediante MCO, se estudiaron los residuos estimados y como se aprecia en la Tabla 11, existían efectos ARCH en los residuos de dicho modelo ${ }^{15}$.

Tabla 11. Estimación de efectos ARCH

\begin{tabular}{|l|c|c|c|c|c|}
\hline Método: MCO & \multicolumn{2}{|c|}{ Variable dependiente: rtcp } & \multicolumn{3}{|c|}{ Muestra: 04/01/2005 - 01/01/2016 } \\
\hline Variable & Coeficiente & ee & t- estadístico & Prob. \\
C & 0,086683 & 0,016194 & 5,352922 & 0,0000 \\
MA(1) & $-0,075088$ & 0,018627 & $-4,031209$ & 0,0001 \\
\hline R $^{2}: 0,005122$ & \multicolumn{1}{|c|}{ JarqueBera (Residuos): 141872,3} & \multicolumn{2}{|c|}{ Prob (JB): 0,0000 } \\
\hline Autocorrelación de residuos & Rezago & Estadístico Q & Prob. (Q) \\
Autocorrelación de residuos $\mathbf{2}$ & 10 & 49,178 & 0,000 \\
& 10 & 320,50 & 0,000 \\
& Rezago & Estadístico X & Prob. $\left(\mathbf{X}^{\mathbf{2}}\right)$ \\
Efectos ARCH & 1 & 261,1925 & 0,0000 \\
Efectos ARCH & 10 & 46,39190 & 0,0000 \\
\hline
\end{tabular}

Fuente: elaboración propia con base en datos dólar Cúcuta. $\alpha=50 \%$.

Prob: probabilidad. Q: estadístico Ljung- Box.

Los modelos de la familia ARCH, mostrados en la Tabla 12, fueron los modelos estimados en este documento; todos ajustaron mediante una distribución t-Student. Los parámetros de cada uno de ellos son estadísticamente significativos y los residuos resultaron ser ruido blanco no gaussiano (ver Apéndice n. $^{\circ} 1$ ), por lo que, concretamente, todos ellos modelan correctamente el comportamiento de los rendimientos del tipo de cambio paralelo de Venezuela. De los modelos de esa tabla, el mejor modelo fue el ARMA $(2,1)$ - EGARCH $(1,1)$, afirmación hecha con base en los criterios de información de Akaike (1974), Schwarz (1978), Shibata (2002) y Hannan-Quinn (1979), pues, reportaron los menores valores de todos

15 Para contrastar la existencia de una estructura $\mathrm{ARCH}$, el mencionado autor propuso un test previo al desarrollo del modelo, conocido como el contraste del multiplicador de Lagrange. Partiendo del sistema de hipótesis $\mathrm{H}_{0}: \alpha_{1}=\alpha_{2}=\ldots \alpha_{q}=0$ (No hay modelo ARCH) y $\mathrm{H}_{1}$ : al menos un $\alpha \neq 0$ (Hay modelo ARCH), toma en consideración los residuos derivados de la estimación por mínimos cuadrados ordinarios (MCO), aproximándose al multiplicador de Lagrange de Breusch y Pagan (1978) y Godfrey (1978) resultando como estadístico de prueba $T \star R^{2} \sim X^{2}{ }^{15}$ 
los modelos. La ecuación de la varianza estimada del modelo seleccionado, representada por la ecuación [18], presentó los signos esperados:

$$
\log (\hat{h})=0,07435+0,91793 \log \left(h_{t-1}\right)+0,13530\left|\frac{\varepsilon_{t-1}}{\sqrt{h_{t-1}}}\right| i+0,9402 \frac{\varepsilon_{t-1}}{\sqrt{h_{t-1}}}
$$

En la ecuación [18], 0,91793 reflejó la persistencia, la volatilidad de la varianza que, según Nelson (1991), forma parte de la varianza heterocedástica. Coeficiente muy cercano a la unidad, en este modelo se expresó por $0,91793 \log \left(h_{t-1}\right)$, lo que significa que el valor estimado de la varianza en el tiempo $t$, arrastra casi por completo la variabilidad del periodo anterior, $t-1$. El efecto asimétrico propio del método, el apalancamiento, fue 0,13530; así, ante una innovación negativa rezagada un periodo, el resultado sobre el logaritmo de la varianza estimada es positivo, mientras que resulta nulo si la innovación referida es positiva. Un choque negativo aumenta la volatilidad, mientras que un choque positivo disminuye la volatilidad. En el caso específico de los rendimientos del tipo de cambio paralelo, un choque negativo se asocia a una depreciación del bolívar frente al dólar, y por el contrario, un choque positivo refiere a una apreciación del mismo.

Se consideró un buen ajuste de la varianza condicional del modelo, como lo reflejó el gráfico (c), de la Figura 5; la varianza estimada replicó correctamente el comportamiento de la volatilidad del tipo de cambio paralelo, como era de esperar. El correlograma cruzado, gráfico (g), entre las observaciones reales y sus cuadrados, ajustó dentro de las bandas, confirmando su aleatoriedad.

La curva de impacto de innovaciones ratificó el efecto asimétrico del modelo EGARCH estimado, gráfico (1) de la Figura 5. Sin embargo, es inconsistente con lo expresado en la ecuación [18], según la afirmación de Nelson (1991), porque el efecto asimétrico que muestra la curva de impacto de innovaciones es positivo para innovaciones positivas y es levemente negativo para innovaciones negativas. Este hecho se podría justificar en la existencia de un cambio de signo en el modelo estimado, que provoca efectos contrarios según el signo de la innovación, pero empíricamente no tiene sentido, porque de ser cierto, una depreciación del tipo de cambio paralelo traería una menor volatilidad, y una apreciación del tipo de cambio estaría relacionada con una mayor volatilidad, y es ampliamente conocido que de esa forma no opera el mercado paralelo de divisas. 
Tabla 12. Síntesis de los modelos de la familia ARCH estimados

\begin{tabular}{|l|c|c|c|c|}
\hline \multicolumn{7}{|l|}{ Modelo: ARCH(3) con modelo de la media ARMA (2,1) } \\
\hline Parámetro & $\boldsymbol{A R}(\mathbf{1})$ & $\boldsymbol{A R}(\mathbf{2})$ & $\boldsymbol{M A ( 1 )}$ & $\boldsymbol{\alpha}_{\mathbf{0}}$ \\
\hline Coeficiente & 0,965190 & 0,032196 & $-0,996025$ & 0,297956 \\
\hline Probabilidad & 0,00000 & 0,00000 & 0,00000 & 0,00000 \\
\hline Parámetro & $\boldsymbol{\alpha}_{\mathbf{1}}$ & $\boldsymbol{\alpha}_{\mathbf{2}}$ & $\boldsymbol{\alpha}_{\mathbf{3}}$ & shape \\
\hline Coeficiente & 0,583422 & 0,227973 & 0,187605 & 2,490772 \\
\hline Probabilidad & 0,00000 & 0,000552 & 0,001151 & 0,000000 \\
\hline Criterios de información & $\mathrm{AIC}=1,787$ & $\mathrm{BIC}=1,8043$ & $\mathrm{~S}=1,7875$ & $\mathrm{HQ}=1,7936$ \\
\hline
\end{tabular}

\begin{tabular}{|c|c|c|c|c|}
\hline \multicolumn{5}{|c|}{ Modelo: GARCH(1,1) con modelo de la media ARMA $(2,1)$} \\
\hline Parámetro & $A R(1)$ & $A R(2)$ & MA(1) & \\
\hline Coeficiente & 0,948912 & 0,031843 & $-0,974580$ & \\
\hline Probabilidad & 0,00000 & 0,00000 & 0,00000 & \\
\hline Parámetro & $\boldsymbol{\alpha}_{0}$ & $\alpha_{1}$ & $\beta_{1}$ & shape \\
\hline Coeficiente & 0,067086 & 0,368870 & 0,630130 & 2,614289 \\
\hline Probabilidad & 0,00000 & 0,00000 & 0,00000 & 0,00000 \\
\hline Criterios de información & $\mathrm{AIC}=1,7705$ & $\mathrm{BIC}=1,7851$ & $\mathrm{~S}=1,7704$ & $\mathrm{HQ}=1,7758$ \\
\hline
\end{tabular}

\begin{tabular}{|c|c|c|c|c|c|c|}
\hline \multicolumn{7}{|c|}{ Modelo: EGARCH $(1,1)$ con modelo de la media ARMA $(2,1)$} \\
\hline Parámetro & $A R(1)$ & $A R$ & & MA(1) & $\boldsymbol{\alpha}_{0}$ & $\alpha_{1}$ \\
\hline Coeficiente & 0,949934 & 0,02 & 185 & $-0,968961$ & 0,074355 & 0,135300 \\
\hline Probabilidad & 0,00000 & 0,00 & & 0,00000 & 0,00029 & 0,015015 \\
\hline Parámetro & \multicolumn{3}{|c|}{$\beta_{1}$} & \multicolumn{2}{|l|}{$\gamma_{1}$} & Shape \\
\hline Coeficiente & \multicolumn{2}{|c|}{0,917931} & \multicolumn{3}{|c|}{0,940224} & 2,10000 \\
\hline Probabilidad & \multicolumn{2}{|c|}{0,00000} & \multicolumn{2}{|r|}{0,00000} & \multicolumn{2}{|c|}{0,00000} \\
\hline Criterios de información & \multicolumn{2}{|c|}{$\mathrm{AIC}=1,7430$} & $\mathrm{BIC}$ & $=1,7598$ & $S=1,7430$ & $\mathrm{HQ}=1,7491$ \\
\hline
\end{tabular}

\begin{tabular}{|l|c|c|c|c|c|}
\hline Modelo: GJR-GARCH(1,2) con modelo de la media ARMA (2,1) \\
\hline Parámetro & $\mathbf{A R ( 1 )}$ & $\boldsymbol{A R ( 2 )}$ & $\boldsymbol{M A}(\mathbf{1})$ & $\boldsymbol{\alpha}_{\mathbf{0}}$ & $\boldsymbol{\alpha}_{\mathbf{1}}$ \\
\hline Coeficiente & 0,949219 & 0,032053 & $-0,974580$ & 0,076510 & 0,503200 \\
\hline Probabilidad & 0,00000 & 0,00000 & 0,00000 & 0,00001 & 0,000000 \\
\hline Parámetro & $\boldsymbol{\beta}_{\mathbf{1}}$ & $\boldsymbol{\beta}_{2}$ & $\gamma_{\mathbf{1}}$ & \multicolumn{2}{|c|}{ shape } \\
\hline Coeficiente & 0,366300 & 0,201994 & $-0,144989$ & 2,610210 \\
\hline Probabilidad & 0,000552 & 0,012595 & 0,06397 & \multicolumn{2}{|c|}{0,00000} \\
\hline Criterios de información & AIC= 1,7689 & BIC= $=1,7878$ & $\mathrm{~S}=1,7689$ & $\mathrm{HQ}=1,7757$ \\
\hline
\end{tabular}




\begin{tabular}{|l|c|c|c|c|c|}
\hline Modelo: APARCH (1,1) con modelo de la media ARMA (2,1) \\
\hline Parámetro & $\boldsymbol{A R}(\mathbf{1})$ & $\boldsymbol{A R}(\mathbf{2})$ & $\boldsymbol{M A}(\mathbf{1})$ & $\alpha_{\mathbf{0}}$ & \\
\hline Coeficiente & 0,949506 & 0,028018 & $-0,970933$ & 0,155222 & \\
\hline Probabilidad & 0,000000 & 0.000000 & 0,000000 & 0,000046 & \\
\hline Parámetro & $\boldsymbol{\alpha}_{\mathbf{1}}$ & $\boldsymbol{\beta}_{\mathbf{1}}$ & $\gamma_{\mathbf{1}}$ & $\delta$ & Shape \\
\hline Coeficiente & 1,000000 & 0,680598 & $-0,116498$ & 1,178105 & 2,10000 \\
\hline Probabilidad & 0,024394 & 0,000000 & 0,000000 & 0,000032 & 0,00000 \\
\hline Criterios de información & AIC $=1,7437$ & BIC=1,7626 & \multicolumn{2}{|c|}{$\mathrm{S}=1,7437$} & $\mathrm{HQ}=1,7505$ \\
\hline
\end{tabular}

Fuente: elaboración propia con base en datos dólar Cúcuta. . AIC: criterio de Akaike. BIC: criterio de Schwarz. S: criterio de Shibata. HQ: criterio de Hannan-Quinn

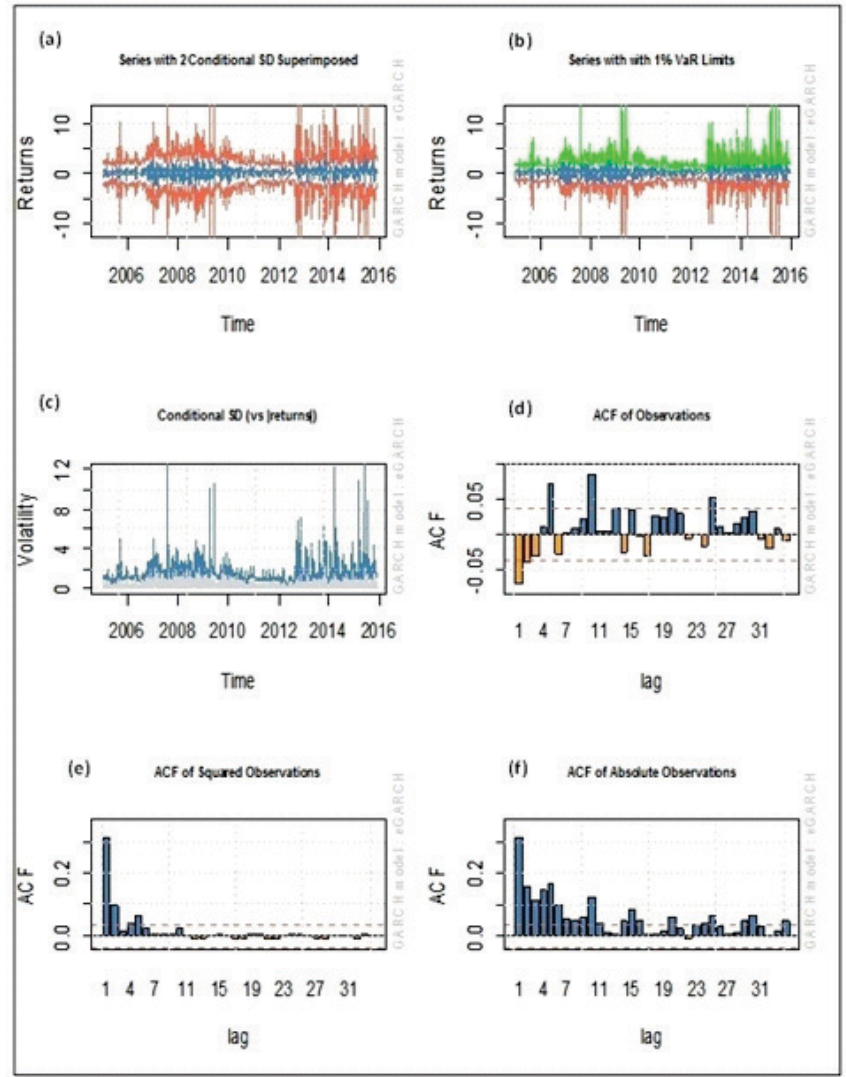

Figura 5. Gráficos de ajuste del modelo ARMA $(2,1)$-EGARCH $(1,1)$.

Fuente: elaboración propia con base en datos dólar Cúcuta. $\alpha=\%$ 


\section{Continuación Figura 5:}

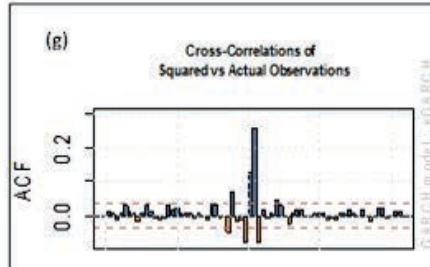

$\begin{array}{lllllllll}-34 & -24 & -14 & -6 & 1 & 7 & 14 & 22 & 30\end{array}$

lag

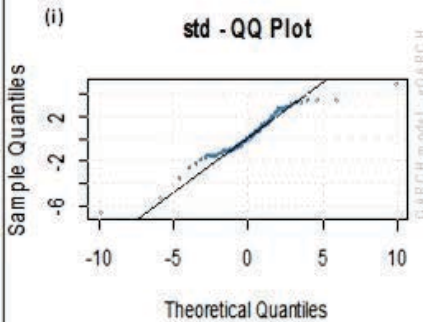

(k)

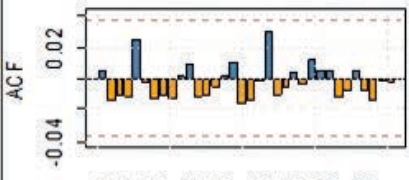

$\begin{array}{lllllllll}1 & 4 & 7 & 11 & 15 & 19 & 23 & 27 & 31\end{array}$

lag

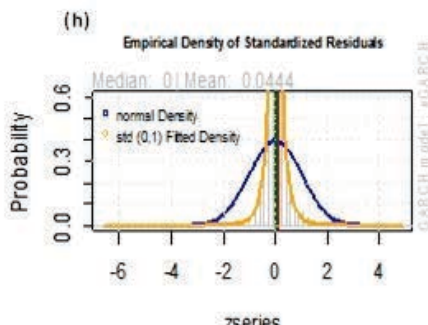

zseries

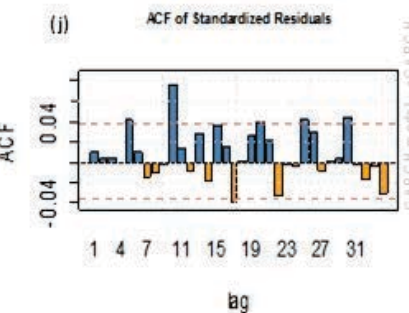

bg

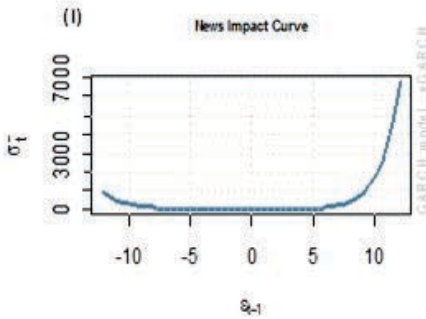

Q-1

Fuente: elaboración propia con base en datos dólar Cúcuta. $\alpha=5 \%$

$\overline{00} \quad$ Incluso dentro de la formación contemporánea de economistas a nivel mundial o asimismo en posgrados relacionados, se ha otorgado mayor importancia al segundo en desmedro del primero. Sin embargo, este será un debate que tendremos que dardesde la academia. 
Los parámetros estimados del modelo ARMA $(2,1)$ - EGARCH $(1,1)$ resultaron no ser estables en conjunto, según el test de Nyblom. Este test prueba la estabilidad simultánea de todos los parámetros, garantiza un buen ajuste para modelos no lineales, deriva un estadístico basado en el multiplicador de Lagrange. La hipótesis nula que maneja es similar a la planteada por el test de estabilidad paramétrica de Chow ( $\mathrm{H}_{\mathrm{o}}$ : todos los parámetros son estables en conjunto). Para el caso del modelo estimado, como se observa en la Tabla 13, el valor calculado del estadístico es mayor que su valor tabulado, garantizando que no hay estabilidad paramétrica conjunta, es decir, los parámetros estimados cambian a lo largo del tiempo, hecho esperado dada la elevada volatilidad de la serie modelada.

En lo que respecta a la estabilidad individual de los parámetros, según el test de Hansen, no todos los parámetros estimados son estables individualmente. La hipótesis nula que maneja el test es que $\beta_{i}{ }^{16}$ es constante $\left(H_{0}: \beta_{i}\right.$ es contante; para $i=1,2, \ldots, k)$. En la Tabla 13 se puede apreciar que los parámetros asociados al modelo ARMA $(2,1)$ de la media sí son estables, en tanto, los correspondientes al modelo EGARCH $(1,1)$ no lo son: $\alpha_{0}$ que representa la mínima varianza, $\alpha_{1}$ que representa el efecto apalancamiento y $\beta_{i}$ que representa la persistencia del modelo no son estables, pero $\gamma_{i}^{\prime}$ asociado al término autorregresivo del error (Nelson, 1991), sí es estable. Este último hecho infiere que, además de ser elevada la persistencia del modelo, esta no es constante y cambia en función del signo de la innovación, arrastrando fuertemente la variabilidad del periodo $t-1$ al periodo $t$. Confirmándose la existencia de conglomerados en la serie, como expone la teoría.

Es importante destacar que estos dos test, Nyblom y Hansen, tienen características similares: i) son fáciles de calcular y son robustos para heterocedasticidad; ii) al igual que el test de Chow, ninguno suministra información sobre el punto de quiebre del parámetro; iii) la distribución del estadístico no es estándar y obedece al valor de $\mathrm{k}$ (número de parámetros estimados), generalmente bajo la hipótesis nula la distribución utilizada es la de Cramer-Von Mises, pero su elección depende de la variable de interés, si es estacionaria o no, o si tiene tendencia determinística y iv) los valores tabulados, para el contraste de hipótesis, fueron calculados por simulación y están codificados en los artículos seminales de los autores.

\footnotetext{
16 No confundir con los parámetros del componente GARCH del modelo, porque en este caso la notación es usada como referencia para indicar cualquier parámetro.
} 
Tabla 13. Test de estabilidad y sesgo de signo del modelo EGARCH estimado

\begin{tabular}{|c|c|c|c|}
\hline \multicolumn{4}{|l|}{ Test de Nyblom } \\
\hline \multicolumn{2}{|l|}{ Valor calculado } & Valor tabulado & Conclusión \\
\hline Estadístico conjunto & 8,01 & 2,11 & No Estables \\
\hline \multicolumn{4}{|l|}{ Test de Hansen } \\
\hline & Valor calculado & Valor tabulado & Conclusión \\
\hline$A R(1)$ & 0,1525 & 0,47 & Estable \\
\hline$A R(2)$ & 0,3075 & 0,47 & Estable \\
\hline MA(1) & 0,1674 & 0,47 & Estable \\
\hline$\alpha_{0}$ & 1,7801 & 0,47 & No estable \\
\hline$\alpha_{1}$ & 0,8326 & 0,47 & No estable \\
\hline$\beta_{1}$ & 1,4276 & 0,47 & No estable \\
\hline$\gamma_{1}$ & 0,2122 & 0,47 & Estable \\
\hline shape & 2,8515 & 0,47 & No estable \\
\hline \multicolumn{4}{|c|}{ Test de sesgo de signo } \\
\hline & & Probabilidad & Conclusión \\
\hline \multirow{3}{*}{\multicolumn{2}{|c|}{$\begin{array}{l}\text { Sesgo en el signo } \\
\text { Sesgo positivo } \\
\text { Sesgo negativo }\end{array}$}} & 0,1121 & No significativo \\
\hline & & 0,9103 & No significativo \\
\hline & & 0,2817 & No significativo \\
\hline
\end{tabular}

Fuente: elaboración propia con base en datos dólar Cúcuta. $\alpha=5 \%$.

En la Tabla 13 también se pueden apreciar los resultados correspondientes al test de sesgo de signo y tamaño. Implica que la varianza condicional depende de forma simultánea del signo y del tamaño de la innovación. En el modelo EGARCH $(1,1)$ estimado, no se encontró evidencia signi- ficativa sobre este tipo de relación en la varianza condicional del modelo estimado, la probabilidad asociada es mayor al nivel de significancia seleccionado. Por ende, el efecto asimétrico sobre la varianza depende del signo de la innovación pero no de su tamaño. 


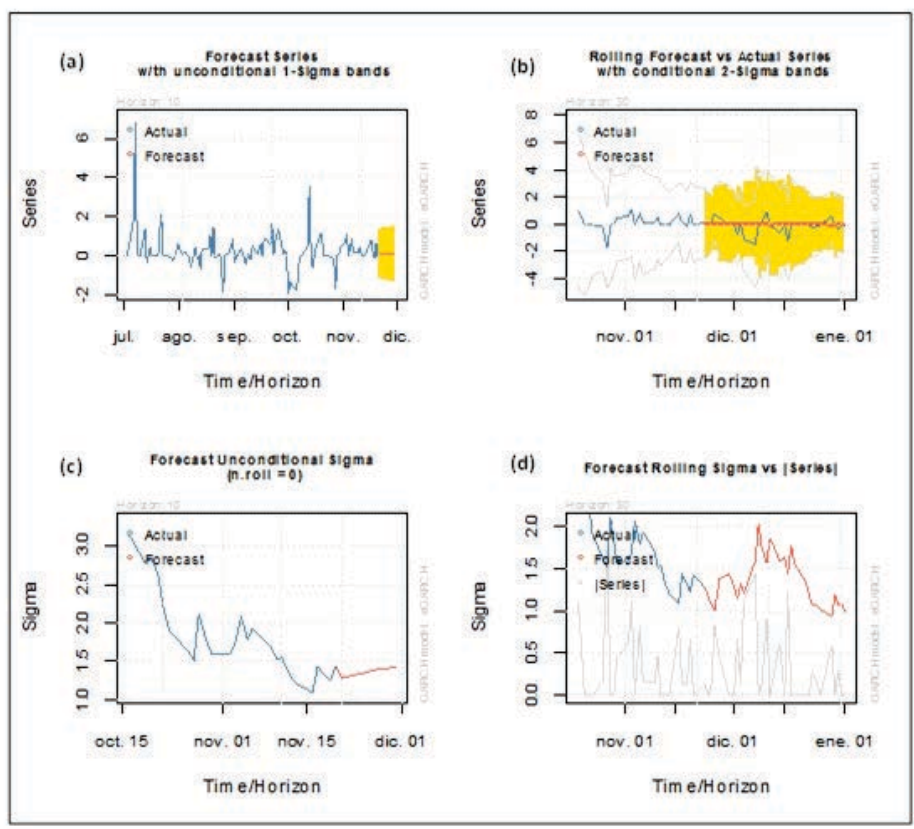

Figura 6. Predicciones del modelo EGARCH estimado.

Fuente: elaboración propia con base en datos dólar Cúcuta.

Finalmente, en cuanto a la bondad en la predicción del modelo, se estimó el modelo dejando 30 observaciones por fuera para hacer los pronósticos. En la Figura 4 se observa que si bien el modelo cumplió con todos los supuestos de validación, sus predicciones no resultaron ser las mejores. En el gráfico (b) de la Figura 6 existe marcada diferencia entre los primeros valores predichos de los rendimientos del tipo de cambio paralelo, a pesar de que, a medida que avanzaba el horizonte temporal, el pronóstico se asemejaba al valor observado de la serie. En el gráfico (d) de la figura referida, la varianza pronosticada no se acercó al valor absoluto de la serie, contradiciendo el pronóstico, el carácter volátil de la serie.

\section{CONCLUSIONES}

Con el fin de modelar el tipo de cambio paralelo de Venezuela entre 2005 y 2015, desde el enfoque de los modelos estocásticos de volatilidad, se realizó una descripción de la variable de interés, considerada una variable próxima al valor real del tipo de cambio paralelo. Se especificó que esta no puede ser utilizada directamente en el proceso de estimación porque no cumple con las características teóricas establecidas. Se construyó la variable rendimientos del 
tipo de cambio paralelo que representa, con mayor precisión, el carácter volátil de la serie original.

En concreto, se identifica que la volatilidad de los rendimientos del tipo de cambio paralelo de Venezuela tiene comportamiento asimétrico, indica que las devaluaciones de la moneda venezolana, producto de las decisiones de política cambiaria, generan un fuerte choque negativo sobre el mercado paralelo de divisas, incrementando la volatilidad del precio del dólar en dicho mercado, como era lo esperado.

En lo que respecta a los modelos estimados, y a la serie de tiempo estudiada, puntualmente se puede decir:

- Los modelos ARIMA no sirven para estimar el comportamiento de los rendimientos del tipo de cambio paralelo debido a la alta volatilidad de la serie, ya que la estructura presente en la serie es no lineal.

- Si bien es cierto que los modelos de la familia ARCH son los adecuados para estimar la variable rendimientos del tipo de cambio paralelo, los modelos utilizados en este estudio no predicen correctamente más de dos observaciones, por lo que no pueden ser empleados como referencia para decisiones relativas a política cambiaria.

- Es importante destacar del párrafo anterior que, a pesar de que los modelos de la familia ARCH no logran predecir los valores de la serie, sí logran modelar correctamente la variable estudiada.

- Como era de esperar, por lo sugerido en la teoría, el modelo que mejor ajustó fue un modelo $\operatorname{EGARCH}(1,1)$. Se confirmó que las series financieras y cambiarias, de alta volatilidad, se modelan mejor cuando se considera la presencia de efectos de apalancamiento.

- El tipo de cambio paralelo de Venezuela entre los años 2005 y 2015, desde el enfoque de las series de tiempo, resulta ser una variable compleja para su modelado. Hecho causado por los factores involucrados en su cálculo; el principal de ellos la incertidumbre, que no es medible directamente, pero que está asociada al incremento del precio del dólar estadounidense en términos del bolívar.

En función de lo antes expuesto se recomienda:

- Cambiar la forma de cálculo de la variable estudiada, porque la estimación del precio del bolívar venezolano en términos del peso colombiano en la frontera no se hace con ninguna rigurosidad estadística, a diferencia de sus otros dos componentes, la oferta monetaria (M2) y las reservas internacionales de Venezuela, que es información tomada de un ente oficial. Esto con el fin de capturar el verdadero comportamiento de la variable.

- Realizar estimaciones con los modelos de la familiar ARCH no empleados en este estudio, para contrastar su ajuste 
y determinar si existe superioridad de alguno de ellos.

- Formalizar estimaciones de modelos de volatilidad estocástica (SV) mediante métodos bayesianos, que precisen el efecto sobre el precio del dólar de variables no observadas.

- Utilizar técnicas multivariantes de análisis de volatilidad para tener mejores opciones en el momento del modelado.

- Dado el crecimiento de la liquidez monetaria y la persistente volatilidad del tipo de cambio paralelo, se sugiere probar la hipótesis del overshooting para la economía venezolana.

\section{REFERENCIAS}

Akaike, H. (1974). A New Look at the Statistical Model Identification. IEEE: Trans. Auto.Control, 19, 719-723.

Arias, F. (2006). El proyecto de investigación. Introducción a la metodología cientifica (5 ed.). Caracas: Episteme.

Baillie, R. (2006). Modelling Volatility, Handbook of Econometrics (Vol. 1). In E. T. a. Patterson (Ed.) New York: Palgrave Macmillan.

Banco Central de Venezuela. (2016). Banco Central de Venezuela. Recuperado de http://www.bcv.org.ve/

Bernanke, B. \& Frank, R. (2007). Principios de economía (3 ed.). Mdrid: McGraw Hill.

Berndt, E., Hall, B., Hall, R. \& Hausman, J. (1974). Estimation lnference in Nonlinear Structural Models. Annals of the Economic and Social Measurement, 4, 653-665.

Bollerslev, T. (1986). Generalized Autoregressive Conditional Heteroskedastic. Journal of Econometrics, (31), 307-327.

Bollerslev, T. \& Wooldrige, J. (1992). Quasi-maximum Likelihood Estimation and Inference in Dynamic Models with Time-Varying Covariances. Econometric Reviews, 11(2), 143-172.

Breusch, T. \& Pagan, A. (1978). A Simple Test for Heterocedasticity and Random Coefficient Variance. Econometrica, 46, 1287-1294. 
Brock, W., Dechert, W., Scheinkman J. \& LeBaron, B. (1996). A Test for Independence Based on the Borrelation Dimension. Econometric Reviews, 3(15), 197-235.

Campbell, A. (1987). Stock Returns and Term Structure. Journal of Financial Economics, 18, 373-399.

Ding, Z., Granger, C. \& Engle, R. (1993). A long memory property of stock market returns and a new model. Journal of Empirical Finance, 83-106.

Dornbusch, R. (1976). Expectations and Exchange Rate Dynamics. The Journal of Political Economy, 84, 1161-1176.

Dornbusch, R., Fischer, S. \& Startz, R. (2009). Macroeconomía (10.a ed.). México, D.F.: McGraw Hill.

Ecoanalítica. (2014, dic.). Entorno y política cambiaria. Caracas. Recuperado de http:// ecoanalitica.com/?wpfb_dl=179

Engle, R. (1982). Autoregressive Conditional Heteroskedasticity with Estimates of the Variance of United Kingdom Inflation. Econometrica, 50(4), 987-1007.

Engle, R. \& Bollerslev, T. (1986). Modelling the Persistence of Conditional Variance. Econometric Reviews, 5, 1-50.

Fama, M. (1963). Risk Returm and Equilibrium: Empirical Test. Jorunal of Financial Economics, 71, 607-636.

Figlewski, S. (1997). Forecasting Volatility. Financial Markets, Institutions and Instruments, 6(1), 2-87.

Glosten, L., Jagannathan, R. \& Runkle, D. (1993). Relationships between the Expected Value and the Volatility of the Nominal Excess Return on Stocks. Northwestern University: Mimeo.

Godfrey, L. (1978). Testing Against General Autoregressive and Moving Average Models when the Regressors include Lagged Dependent Variables. Econometrica, 46, 1294-1302.

Hansen, B. (1992). Tests for Parameter Instability in Regressions with I(1) Processes. Journal of Business and Economic Statistics, 10, 321-336. 
Hansen, P. \& Lunde, A. . (2005). A forecast comparison of volatility models: does anything beat a GARCH $(1,1)$ ? Journal of Applied Econometrics, 20, 873-889.

Harvey, A. (1981). The Econometric Analysis of Time Series. Oxford: Phillip Alan.

Hentschel, L. (1995). All in the Family Nesting Symmetric and Asymmetric GARCH Models. Journal of Financial Economics, 39, 71-104.

Hsieh, D. (1995). Nonlinear Dynamics in Financial Markets Evidence and Implications. Duke: Institute for Quantitative Research in Finance.

Mandelbrot, B. (1963). The Variation of Certain Speculative Prices. Journal of Business, 36, 394-419.

Márquez, M. (2002). Modelo setar aplicado a la volatilidad de la rentabilidad de las acciones: algoritmos para su identificación. Tesis de Maestría en Estadística. Universitat Politècnica de Catalunya, Barcelona.

McLeod, A. \& Li, W. (1983). Diagnostic Checking ARMA Time Series Models Using Squared Residual Autocorrelations. Journal of Time Series Analysis, 4, 269-273.

Milhoj, A. (1987). A Multiplicative Parametrization of ARCH Models. Research Report 101. Copenhagen: Institute of Statistics, University of Copenhagen.

Nelson, D. B \& Cao, C. Q. (1992). Inequality Constraints in the Univariate GARCH Model. Journal of Business \& Economic Statistics, 10, 229-235.

Nelson, D. B. (1991). Conditional Heterocedasticity in Asset Returns: A New Approach. Econometrica, 59, 347-370.

Nyblom, J. (1989). Testing for the Constancy of Parameters Over Time. Journal of the American Statistical Association, 84(405), 223-230.

Poon, S. \& Granger, C. (2003). Forecasting Volatility in Financial Markets: A Review. Journal of Economic Literature, 41, 478-539.

Poterba, J. \& Summers, L. (1986). The Persistence of Volatility and Stock Market Fluctuations. American Economic Review, 76, 1142-1151. 
República Bolivariana (2014, 20 de feb.). Decreto con Rango Fuerza y Valor de Ley del Régimen Cambiario y sus Ílicitos. Gaceta Oficial de la República Bolivariana, (6.126). Recuperado de www.tsj.gob.ve/gaceta-oficial

Samuelson, P. \& Nordhaus, W. (2010). Economía con aplicaciones a Latinoamérica (19 ed.). México D.F.: McGraw Hill.

Sánchez, A. \& Reyes, M. (2006). Regularidades probabilísticas de las series financieras y la familia de modelos GARCH. Ciencia Ergo Sum, 13(2), 149-156.

Schwarz, G. (1978). Estimating the Dimension of a Model. Annals of Statistics, 6, 461-464.

Shibata, R. (2002). Information Criteria for Statistical Model Selection. Electron. Comm. Jpn. Pt. III, 85, 32-38.

Taylor, S. (1986). Modelling Financial Time Series. New York: John Wiley.

The Comprehensive R Archive Network -CRAN-. (2016, 20 de jul.). The R Project for Statistical Computing. Retrieved from http://cran.r-project.org/manuals.html

Tsay, R. (1986). Nonlinearity Test for Time Series. Biometrika, 76, 461-466.

Zakoian, J. (1990). Threshold Heteroskedastic Model. Paris: INSEE Mimeo. 
Anexo 1. Diagnóstico de los residuos de los modelos estimados

\begin{tabular}{|c|c|c|c|c|c|c|}
\hline \multicolumn{7}{|c|}{ Modelo: $\mathrm{ARCH}(3)$ con modelo de la media ARMA $(2,1)$} \\
\hline & Mediana & Media & Varianza & $\begin{array}{c}\text { Error } \\
\text { estándar }\end{array}$ & Asimetría & Kurtosis \\
\hline Residuos (R) & $-0,0102$ & 0,0945 & 1,0805 & 1,0395 & 2,2280 & 52,4957 \\
\hline Shapiro-Wilk (R) & & 0,7511 & \multicolumn{2}{|c|}{$\operatorname{Prob}[\mathrm{S}-\mathrm{W}(\mathrm{R})]$} & $<2.2 \mathrm{e}-16$ & \\
\hline Shapiro-Wilk $\left(\mathrm{R}^{2}\right)$ & & 0,0827 & \multicolumn{2}{|c|}{ Prob[S-W(R2)] } & $<2.2 \mathrm{e}-16$ & \\
\hline Ljung-Box(R) & & 0,96641 & \multicolumn{2}{|c|}{ Prob[L-B(R)] } & 0,3256 & \\
\hline Ljung-Box $\left(\mathrm{R}^{2}\right)$ & & 0,05164 & \multicolumn{2}{|c|}{ Prob[L-B(R2)] } & 0,8202 & \\
\hline Efectos ARCH (LM) & & 8,0977 & \multicolumn{2}{|c|}{ Prob[L-M] } & 0,7774 & \\
\hline
\end{tabular}

\begin{tabular}{|l|c|c|l|c|c|c|}
\hline \multicolumn{6}{|c|}{ Modelo: GARCH(1,1) con modelo de la media ARMA (2,1) } \\
\hline & Mediana & Media & Varianza & $\begin{array}{c}\text { Error } \\
\text { estándar }\end{array}$ & Asimetría & Kurtosis \\
\hline Residuos (R) & $-0,0057$ & 0,0964 & 1,1236 & 1,0600 & 0,2378 & 28,6771 \\
\hline Shapiro-Wilk (R) & & 0,7898 & Prob[S-W(R)] & $<2.2 \mathrm{e}-16$ & \\
\hline Shapiro-Wilk (R $\left.{ }^{2}\right)$ & & 0,1332 & Prob[S-W(R $\left.\left.{ }^{2}\right)\right]$ & $<2.2 \mathrm{e}-16$ & \\
\hline Ljung-Box(R) & & 0,44873 & Prob[L-B(R)] & 0,5029 & \\
\hline Ljung-Box(R $\left.{ }^{2}\right)$ & & 0,11783 & Prob[L-B(R $\left.\left.{ }^{2}\right)\right]$ & 0,7314 & \\
\hline Efectos ARCH (LM) & & 4,5966 & Prob[L-M [ & 0,9701 & \\
\hline
\end{tabular}

\begin{tabular}{|l|c|c|l|l|l|l|}
\hline \multicolumn{6}{|c|}{ Modelo: EGARCH(1,1) con modelo de la media ARMA (2,1) } \\
\hline & Mediana & Media & Varianza & $\begin{array}{c}\text { Error } \\
\text { estándar }\end{array}$ & Asimetría & Kurtosis \\
\hline Residuos (R) & $-0,00272$ & 0,04448 & 0,242307 & 0,492247 & 0,513172 & 25,00642 \\
\hline Shapiro-Wilk (R) & & 0,7945 & Prob[S-W(R)] & $<2.2 \mathrm{e}-16$ & \\
\hline Shapiro-Wilk (R $\left.{ }^{2}\right)$ & & 0,1482 & Prob[S-W(R $\left.\left.{ }^{2}\right)\right]$ & $<2.2 \mathrm{e}-16$ & \\
\hline Ljung-Box(R) & & 0,53262 & Prob[L-B(R)] & 0,4655 & \\
\hline Ljung-Box(R $\left(\mathrm{R}^{2}\right)$ & & 0,03404 & Prob[L-B(R $\left.\left.{ }^{2}\right)\right]$ & 0,8536 & \\
\hline Efectos ARCH (LM) & & 6,0061 & Prob[L-M $]$ & 0,9158 & \\
\hline
\end{tabular}

\begin{tabular}{|l|c|c|l|c|c|c|}
\hline \multicolumn{5}{|c|}{ Modelo: GJR-GARCH(1,2) con modelo de la media ARMA (2,1) } \\
\hline & Mediana & Media & Varianza & $\begin{array}{c}\text { Error } \\
\text { estándar }\end{array}$ & Asimetría & Kurtosis \\
\hline Residuos (R) & $-0,0064$ & 0,0932 & 1,087327 & 1,04275 & 0,32399 & 25,4524 \\
\hline Shapiro-Wilk (R) & & 0,8003 & Prob $[S-W(R)]$ & $<2.2 \mathrm{e}-16$ & \\
\hline Shapiro-Wilk (R $\left.{ }^{2}\right)$ & & 0,1448 & Prob[S-W(R $\left.\left.{ }^{2}\right)\right]$ & $<2.2 \mathrm{e}-16$ & \\
\hline Ljung-Box(R) & & 0,37367 & Prob[L-B(R)] & 0,541 & \\
\hline Ljung-Box(R $\left.{ }^{2}\right)$ & & 0,02645 & Prob[L-B(R $\left.\left.{ }^{2}\right)\right]$ & 0,8708 & \\
\hline Efectos ARCH (LM) & & 4,6775 & Prob $[\mathrm{L}-\mathrm{M}]$ & 0,9679 & \\
\hline
\end{tabular}




\begin{tabular}{|l|c|c|l|c|c|c|}
\hline \multicolumn{6}{|c|}{ Modelo: APARCH (1,1) con } & \multicolumn{1}{c|}{ modelo de la media ARMA (2,1) } \\
\hline & Mediana & Media & Varianza & $\begin{array}{c}\text { Error } \\
\text { estándar }\end{array}$ & Asimetría & Kurtosis \\
\hline Residuos (R) & $-0,0026$ & 0,04678 & 0,25599 & 0,50596 & 0,62847 & 27,2023 \\
\hline Shapiro-Wilk (R) & & 0,7758 & Prob[S-W(R)] & $<2.2 \mathrm{e}-16$ & \\
\hline Shapiro-Wilk (R $\left.{ }^{2}\right)$ & & 0,1425 & Prob[S-W(R $\left.\left.{ }^{2}\right)\right]$ & $<2.2 \mathrm{e}-16$ & \\
\hline Ljung-Box(R) & & 0,0177 & Prob[L-B(R)] & 0,8939 & \\
\hline Ljung-Box(R $\left(\mathrm{R}^{2}\right)$ & & 0,0792 & Prob[L-B(R $\left.\left.{ }^{2}\right)\right]$ & 0,7783 & \\
\hline Efectos ARCH (LM) & & 8,3436 & Prob[L-M] & 0,7577 & \\
\hline
\end{tabular}

Nota: nivel de significancia $=5 \%$ 


\section{Anexo 2. Método de cuasi máximo verosimilitud}

Suponiendo que sea $\left\{\left(y_{t}, z_{t}\right): t=1,2, \ldots\right\}$ una secuencia de vectores aleatorios, tal que, $y_{t}$ de orden $\mathrm{k}_{\mathrm{x}} 1$ contiene las variables endógenas $\mathrm{y}_{t}$ de orden $\mathrm{L}_{\mathrm{x}} 1$, contiene las variables exógenas. Sea $x_{t}=\left(z_{t}, y_{t-1}, z_{t-1}, \ldots, y_{1}, z_{1}\right)$, un vector de variables predeterminadas, entonces, el propósito es estimar los dos primeros momentos condicionales de $y_{t}$ dado los valores de $x_{t} \cdot{ }^{17}$

La media y la varianza condicionales son conjuntamente parametrizadas por un vector $\theta$ de dimensión finita, y se definen:

$$
\begin{gathered}
\left\{\mu_{t}\left(x_{t}, \theta\right): \theta \in \Theta\right\} \\
\left\{\sigma_{t}^{2}\left(x_{t}, \theta\right): \theta \in \Theta\right\}
\end{gathered}
$$

donde, $\Theta$ es un subconjunto de $\mathbf{R}, \mu_{t} \mathrm{y} \sigma_{t}^{2}$ son funciones conocidas de $x_{t} \mathrm{y} \theta{ }^{18}$

Entonces, el logaritmo condicional de la cuasi verosimilitud está dado por:

$$
\ell\left(\theta, y_{t}, x_{t}\right)=-1 / 2 \log \left|\sigma_{t}^{2}\left(x_{t}, \theta\right)\right|-1 / 2\left(y_{t}-\mu_{t}\left(x_{t}, \theta\right)\right)^{\prime} \sigma_{t}^{2}\left(x_{t}, \theta\right)^{-1}\left(y_{t}-\mu_{t}\left(x_{t}, \theta\right)\right)
$$

donde, $\varepsilon_{t}\left(y_{t}, x_{t}, \theta\right) \equiv\left(y_{t}-\mu_{t}\left(x_{t}, \theta\right)\right)$, denota los residuos de la función $\mathrm{y}$, si se suprime la dependencia entre $\varepsilon_{t} \mathrm{y} \sigma_{t}^{2}, y_{t} \mathrm{y} x_{t}$ la expresión del logaritmo de la cuasi verosimilitud puede redefinirse como:

$$
\ell(\theta)=-1 / 2 \log \left|\sigma_{t}^{2}(\theta)\right|-1 / 2\left(\varepsilon_{t}\right)^{\prime} \sigma_{t}^{2}(\theta)^{-1}\left(\varepsilon_{t}(\theta)\right)
$$

Y por definición el estimador CMV es:

$$
L_{T}(\theta)=\Sigma_{t-1}^{\mathrm{T}} \ell_{t}(\theta)
$$

\footnotetext{
Si el análisis se quiere hacer solo para los valores rezagados de $y_{t}, z_{t}$ puede ser excluido sin alterar ninguna afirmación posterior.

18 Así $E\left(y_{t} \mid x_{t}\right)=\mu_{t}\left(x_{t}, \theta\right)$ y $\operatorname{Var}\left(y_{t} \mid x_{t}\right)=\mu_{t}\left(x_{t}, \theta\right)$ para t: $1,2, \ldots$
} 
Anexo 3. Algoritmo Berndt, Hall, Hall y Hausman

Básicamente, si la estructura del modelo se supone:

$$
F_{t}\left(y_{t}, \beta\right)=\varepsilon_{t}
$$

donde, $y_{t}$ es un vector fila de orden $1_{x} \mathrm{~m}$ que contiene las variables dependientes, $F_{t}$ es un función dos veces diferenciable cuyo valor es un vector $1_{\mathrm{x}} \mathrm{m}, \beta$ es un vector de parámetros desconocidos de orden $\mathrm{k}_{\mathrm{x}} 1$ y $\varepsilon_{t}$ es un vector de perturbaciones aleatorias, que se distribuyen de acuerdo a una normal multivariante, con media cero y matriz de varianza y covarianzas $\Sigma$.

La estimación de $\beta$ se puede hacer por dos vías: i) máxima versimilitud y ii) distancia mínima $(\mathrm{DM})^{19}$. Lo que respecta a la $\mathrm{MV}$, es suficientemente conocido en el trabajo econométrico. La distancia mínima es frecuentemente utilizado en el trabajo práctico para la estimación simultánea de modelos de estructuras lineales ${ }^{20}$. La ventaja de MV sobre DM es que los estimadores que arroja el último no son eficientes, generalmente.

Berndt, Hall, Hall y Hausman, proponen maximizar la función escalar $V(x)$, de un vector $x$ de longitud $k$; asumen que $\mathrm{V}$ es dos veces diferenciable y continua, además tiene conjuntos compactos de nivel superior. Parte su análisis de un teorema:

Teorema del Gradiente: Considere el gradiente de V en $x, g=\partial v(x) / \partial x$. Entonces cualquier vector d, en el mismo espacio medio de $g$ (es decir, $d^{\prime} g>0$ ) está en dirección de incremento de $V(x)$, en el sentido de que $V(x+\lambda d)$, es un incremento de la función del escalar $\lambda$, al menos lo suficientemente pequeño de tamaño $\lambda$.

El éxito del algoritmo es que elige una dirección en cada iteración, que se encuentra en el espacio medio definido por el gradiente. En otras palabras, el método busca calcular el gradiente $g$, derivado de una dirección $d$, y después encontrar el valor de $\lambda$ que maximice $V(x+\lambda d)$, garantizando convergencia.

El conjunto de direcciones de $d$, puede ser derivado de multiplicar el gradiente por un número definido positivo, llamado Q. En estadística, generalmente, Q es la matriz de varianzas y covarianzas de las estimaciones; es necesario, que Q no se aproxime

$\overline{19}$ A diferencia del algoritmo de Newton que requiere mayor rigurosidad matemática, el $\mathrm{BHHH}$ garantiza convergencia con menor rigor de cálculo

20 Los estimadores mínimos cuadrados en tres etapas son un ejemplo. 
a una matriz singular, para ello se define una restricción: sea $\alpha$ una constante positiva menor que uno, en cada iteración se requiere:

$$
r=\frac{d^{\prime} g}{d^{\prime} d}>\alpha
$$

Si $r<\alpha$, en una iteración particular, se puede reemplazar Q por una matriz con grandes elementos en la diagonal principal. La restricción siempre puede ser satisfecha si Q es igual a la matriz identidad, $(\mathrm{Q}=\mathrm{I})$ que es perfectamente aceptable.

El reto del método está en seleccionar el $\lambda$. Sea $\delta$ una constante prestablecida en el intervalo $(0,1 / 2)$, se define:

$$
\gamma(x, d)=V(x+\lambda d)-V(x) / \lambda d^{\prime} g
$$

Si $\gamma(x, d) \geq \delta, \lambda=1$; de lo contrario, $\delta \leq \gamma(x, d) \leq 1-\delta$.

Una vez definido el valor de $\lambda$, la convergencia del algoritmo está garantizada. 